\title{
A New Conceptual Framework for the Therapy by Optimized Multidimensional Pulses of Therapeutic Activity. The case of Multiple Myeloma Model.
}

\author{
Denis Horvath \\ Technology and Innovation Park, Centre of Interdisciplinary Biosciences, \\ Faculty of Science, P. J. Šafárik University, \\ Jesenná 5, 04154 Košice, Slovak Republic \\ horvath.denis@gmail.com \\ Branislav Brutovsky \\ Department of Biophysics, Faculty of Science, P. J. Šafárik University, \\ Jesenná 5, 04154 Košice, Slovak Republic \\ branislav.brutovsky@upjs.sk
}

March 27, 2022

\begin{abstract}
We developed simulation methodology to assess eventual therapeutic efficiency of exogenous multiparametric changes in a four-component cellular system described by the system of ordinary differential equations. The method is numerically implemented to simulate the temporal behavior of a cellular system of multiple myeloma cells. The problem is conceived as an inverse optimization task where the alternative temporal changes of selected parameters of the ordinary differential equations represent candidate solutions and the objective function quantifies the goals of the therapy. The system under study consists of two main cellular components, tumor cells and their cellular environment, respectively. The subset of model parameters closely related to the environment is substituted by exogenous time dependencies - therapeutic pulses combining continuous functions and discrete parameters subordinated thereafter to the optimization. Synergistic interaction of temporal parametric changes has been observed and quantified whereby two or more dynamic parameters show effects that absent if either parameter is stimulated alone. We expect that the theoretical insight into unstable tumor growth provided by the sensitivity and optimization studies could, eventually, help in designing combination therapies.
\end{abstract}

Keywords: multiple myeloma, tumor and environment, osteoblast and osteoclast populations, ordinary differential equations, minimax optimization, sensitivity analysis 


\section{Introduction}

Mathematical models of cancer are widely used to get insight into dynamics of cancer initiation and progression whereby they help researchers to design new therapeutical strategies [1, 2, 3, 4]. As a consequence of ongoing accumulation of relevant biological knowledge, as well as availability of prognostic variables, complexity of mathematical models in tumor biology constantly increases. At the same time, the bottom-up derivation of the relation between microscopic level and macroscopic behavior is far from straightforward. The balance between biological relevance and mathematical complexity is typically achieved by means of iterations containing addition or omission of algebraic expressions in the respective model. Despite the vast majority of iterative model-modifying operations is motivated by relatively well understood short-term effects of the respective variables and algebraic terms, too many details can, paradoxically, make the overall interpretation of the numerical results more difficult [5].

Due to nonlinear interactions, that pose the most difficult obstacle (except the fixed points), the long-term or large-scale outcomes cannot be usually interpreted without detailed simulations. Along with progress in oncological experimental studies and synthesizing of available information, emerging field of multi-scale modeling [6] provides novel computational strategies of exploring cancer biology simultaneously with anticancer therapies. Although this type of approach enables to reduce the number of degrees of freedom, the problem associated with too many parameters and their relevance persists, which is the common problem in most cancer simulation models and methodologies, thus requiring to address the issue of parametric uncertainty, unidentifiability and relevance [5, 7].

Motivated by these general unresolved questions of parametric importance, we developed the specific theoretical approach based on the model of bone remodeling by Komarova et al. [8] and its phenomenological extension applied to multiple myeloma (MM) bone disease [9]. The above models illustrate the association of incremental incorporation of novel biological information with an increase in the number of the parameters. It follows that to control particular scenario (outcome) by the parameters of the model, it is essential to estimate the relevance of the specific parameters and their meaningful groupings. The issue of parametric relevance is especially challenging from the point of view of the therapy design. Within the context of mathematical models of cancer or tumor growth, there is a systematic effort towards identification of the appropriate parameters in order to achieve, hopefully, therapeutic effects by parametric modifications [1].

The leitmotiv of here proposed methodology consists in the idea that coordinated multiparametric (highdimensional) changes can positively, in a therapeutic sense, effect cell populations. Most of the previous theoretical studies of cancer have focused primarily on the virtual therapeutic interventions performed via variations of parameters which describe drug-induced proliferation, necrosis or apoptosis (see Ref.[1] and references therein). Since non-linear systems, including the cancer models [10], show a wide range of emerging and unstable behaviors, we assume that not yet fully understood area of therapeutic options can be much more fruitful and more structured than intuitively expected [11].

To overcome the existing barriers to treatment, such as the resistance to chemotherapy, it seems important to explore more advanced and universal strategic options (see e.g. [12, 13, 14]). Deeper understanding of their impact might be achieved by combining knowledge from complementary research fields, such as optimization theory, inverse problems [15], and sensitivity analysis [16, 17, 18, 19, 20, 21, 22, 23, 24]. 
In the present study we pursue the idea of multidimensional investigation of the global parametric sensitivity of cancer population models. Our method extends the range of methods for the sensitivity analysis of the systems of nonlinear ordinary differential equations (ODEs). Therefore, instead of application of standard analysis of the respective ODEs system, we are interested in the specific global parametric sensitivity analysis [23] which can be seen as a way to propose a qualitatively new therapies. Despite obvious differences in the technical and implementation details, simultaneous use of the optimization and sensitivity analysis is commonly applied feature [16, 19].

To put forward the above ideas, we apply them to analyze MM bone disease. In healthy bone tissue, the bone homeostasis is maintained (or re-established after fracture or microscale damages) by many coordinated actions at cellular and molecular levels, summarily called the bone remodeling [25]. The process maintains the balance between removal of the bone tissue (resorption) and the formation of new tissue (ossification). The bone remodeling involves two crucial cell types - osteoclasts (OCs) and osteoblasts (OBs). The former cells are responsible for disassembling the old bone tissue, the latter for synthesizing the new tissue. The imbalance between intensities of the two processes leads to bone diseases, such as osteoporosis or bone cancer. Physiological numbers of OCs and OBs are guaranteed by their mutual influence via autocrine and paracrine signaling. Due to serious health implications of the MM cancer, several mathematical models were developed to understand nonlinear dynamics of the OCs and OBs production, homeostasis and decay [26, 27, 28, 29, 30], proposing alternative equations to describe the system. The assembly composed of many coupled submodels [31] has been considered to adequately describe calcium homeostasis and intracellular signaling as occurring in the process of bone remodeling. The analysis of homeostatic control also means a better understanding of MM, which is often manifested by hypercalcemia [32].

Here presented computational considerations derive from the ODEs model by Komarova et al. [8] describing population dynamics of OCs and OBs in healthy tissue. Their parametrization of the net effectiveness of the OCs and OBs autocrine and paracrine factors, such as TGF- $\beta$ (one of the classes of polypeptide transforming growth factors), RANKL (the receptor activator of nuclear factor kappa-B ligand), and OPG (osteoclastogenesis inhibitory factor), was used in the recent mathematical model of multiple myeloma by Koenders and Saso [9]. Therein, the authors augmented the above model by Komarova et al. [8] by including the population of myeloma cells with the feedback to the OCs proliferation and the OBs decay into the model. Moreover, the population of so-called joint cells (JCs) also called OCs-MM hybrid cells [33, 34], formed when one OC and one MM cell meet, was included into the extended set of equations [9]. This model also includes the tumoral feedback on the paracrine interactions, interactions of OBs and OCs [35]. The OCs-MM hybrid cells co-cultures are considered to contribute significantly to the formation of bone-resorbing OCs and bone destruction in the MM case. Although widely discussed phenomena of intratumoral MM heterogeneity [30, 36], phenotypic plasticity [29] and drug resistance can decisively influence the treatment, we leave these aspects to further research. On the other hand, formation of JCs belonging to the wide class of pathological cell fusion processes in cancer [37, 38], already captured at some basic level in [9], can be considered as an alternative mechanism contributing to the phenotypic heterogeneity thereby increasing the chemical resistance and metastatic potential. Promising approach to the MM analysis represents the agent based model introduced in [29] which incorporates DKK1-Wnt-OPG/RANKL pathway and cytokine stimulation. The main strength of the model consists in the study of therapeutic effects of Lidamycin, glucocorticoids and Anti-DKK1 mAb (BHQ880). Unfortunately, it is rather difficult to compare or 
associate this type of models to ODEs.

In the present work we address the problems discussed in [9] from different perspectives and research interests. The emphasis is placed on the specific aspects of the sensitivity analysis, parametric relevance, and optimization of therapeutic interventions. Here applied variant of the sensitivity analysis uses therapeutically relevant parametric moves (pulses) determined as the worst-case optimization outputs.

To develop the efficient therapeutic schedule, one implicitly faces to several, sometimes conflicting [39] or overlapping, objectives which must be considered simultaneously. Regarding this, our motivation for the optimization of parametric combinations is, apart from the search for the efficient solution, to formulate the methodological multi-objective framework that may provide basis for further studies. Despite intuitive plausibility of the multi-objective formulation, solutions of the majority of multi-objective problems are not straightforward, as different paradigms often lead to different solutions. There is a variety of methods how to quantify the quality of the respective solution which is expected to fulfill simultaneously a few objectives [40]. Among them, the scalarization approach [41] which leads to a compromise single-objective formulation is one of the widely used practices. From the point of view of the reliability of the results, the degree of consistency (stability) achieved through diverse scalarizations is important. In the most favorable circumstances, the scalarization points to the single solution on the Pareto front.

In our paper, in addition to detailed analysis of the worst-case "minimax" scalarization, we present the alternative numerical experiments with the optimization of single-objective functions based on the aggregation transforms of several objectives [42]. In order to avoid uncertainty in the interpretation of our results, we use optimization that does not use stochastic sampling and is intentionally limited to generating deterministic outputs. To analyze the respective non-differentiable scalarizations, we employed the comprehensive direct grid search which evaluates the pair of purposefully constructed objective functions at each grid point of some feasible parametric region. This choice reflects the recent trends in machine learning [43, 44] where the grid search variants also known as hyperparameter searches receive increasing attention.

The main aim of our study is to bring interesting alternative approach which could stimulate further research in the respective direction instead of demonstrating superiority of the specific optimization algorithm. Systematic analysis performed by the grid search technique can help to find parametric boundaries within which improvements can be done (even if highly diluted grids are used). We note that, at this stage, the independence of the grid search technique on the initial conditions with no tendency of being trapped in a local optimum vindicates its (low) computational performance (nevertheless, in sec. 6.1 we discuss the hybrid discrete-continuous optimization methodology which has significant potential to improve the coarse grid results).

The theoretical background to our considerations is the four cell population model [9] described by the system of four ODEs written in the normal form

$$
\begin{aligned}
\frac{d C}{d t} & =R_{C}(T, C, B), & \frac{d B}{d t} & =R_{B}(T, C, B), \\
\frac{d T}{d t} & =R_{T}(C, T, J), & \frac{d J}{d t} & =R_{J}(C, T, J),
\end{aligned}
$$

where the respective rates of change of the four populations, $C, B, T, J$, referring to the population of OCs $(C)$, 
OBs $(B), \mathrm{MM}(T)$ and JC $(J)$, respectively, have the explicit form

$$
\begin{aligned}
R_{C} & \equiv \alpha_{C}\left(1+h_{C T} T\right) C^{g_{C C}} B^{g_{C B}}-\beta_{C} C-\alpha_{J} C T \\
R_{B} & =\alpha_{B}\left(1-h_{B T} T\right) C^{g_{B C}} B^{g_{B B}}-\beta_{B} B \\
R_{T} & \equiv \alpha_{T} C^{g_{T C}} T^{g_{T T}}-\beta_{T} T-\alpha_{J} C T+\kappa_{J} J \\
R_{J} & \equiv \alpha_{J} C T-\beta_{J} J .
\end{aligned}
$$

This autonomous system couples population number of the OCs, MM cells, and JCs in the representative volume of bone marrow basic multicellular units (BMU); $\alpha_{\bullet}, \beta_{\bullet}$ represent activities of the cell production and removal. The power-law nonlinear structure proposed by [8] of the interactions is parametrized by $g_{\bullet \bullet}$ which represent the effectiveness of the OCs and OBs autocrine and paracrine factors. Increased sensitivity of OCs sand OBs cells due to the influence of MM cells is modeled by $h_{C T}$ parameter. The interpretation behind the term $h_{C T} T C^{g_{C C}} B^{g_{C B}}$ is that $\mathrm{MM}$ cells stimulate bone metabolism and bone marrow micro-environment by means of RANKL, decrease in OPG expression and production of chemokines MIP-1 $\alpha$ (human macrophage inflammatory protein), MIP $-1 \beta$ (macrophage inflammatory protein-1), and SDF-1 $\alpha$ (stromal cell-derived factor) [9, 45]. Furthermore, the term $\left(-h_{B T}\right) T C^{g_{B C}} B^{g_{B B}}$ describes how MM cells suppress OB function by the secretion of OB inhibiting factors, such as Wnt inhibitors DKK -1 and sFRP -2 . The parameter $\kappa_{J}$ in the term $\kappa_{J} J$ denotes the backward transformation rate of the JCs into MM cells with the specific assumption [9] that while MM cells survive dissociation, OCs are not recreated, which means that there is no adequate term $\propto J$ in the $R_{C}$ [9].

The paper is organized as follows. In the section 2 we introduce basics of the methodology including the parametrization of symmetric pulses and the form of the objective function. In the section 3 we present numerical results obtained for given parametric settings. The alternative scalarizations of the multi-objective problem are discussed in sec4. In section 5 the alternative objective function with the regularization penalty term to minimize toxic side effects (at some stylized level) of the therapies is studied. The model of the more realistic exogenous asymmetric therapeutic pulses is introduced in sec. 6, where we also provide extensive comparison of the impact of symmetric and asymmetric pulse forms, respectively, on the optimum obtained. In addition, there are presented results of the optimization performed on the selected manifolds (which go beyond the results provided by grid search). The generalization of synergistic quantification of the parametric pairs is presented as well. Finally, the conclusions are presented. Two appendices provide additional information about the robustness of optima in periodic environments and numerical accuracy of the calculations.

\section{The methodology}

In this section we provide methodological details of our approach. The main aim of our work is to formalize and analyze the time-varying influence of the populations of OCs and OBs (viewed as the environment) on the populations of tumoral cells (including the joint cells) via autocrine and paracrine interactions and, consequently, to exploit this framework to drive dynamics of the tumor cells population towards required direction. 


\subsection{The equilibrium approximation and short-time dynamics}

Without sufficient knowledge of symmetry or invariance, or without significant simplifications the numerical solution is, in principle, the only universal option to study population dynamics (Eqs. 1) under very general nonlinear conditions. Nevertheless, some partial insights into the tumor behavior can be achieved without actually solving the corresponding ODEs numerically by starting with the static equilibrium analysis and then proceeding with approximate dynamic considerations.

The information about equilibrium enables to derive long-term trends in overall dynamics. Although the system of the transcendental equations $R_{X}=0 ; X \in\{C, B, T, J\}$ with $R_{X}$ given by Eq. (2) presents no computational difficulty, the problem is that the system does not provide steady-state solutions (fixed points) in the explicit form. Analytical solvability can be achieved exclusively for the choice $g_{T C}=0$ and $J=0$ [28, 9]. Discussion about the stability is postponed to the subsection 3.1.1. Despite its biological limitations, the above approximation is useful as an initial guess for more advanced formula. In such case the equilibrium solution may be expressed as

$$
\begin{aligned}
T_{e q}^{(0)} & =\left(\frac{\alpha_{T}}{\beta_{T}}\right)^{\frac{1}{1-g_{T T}}}, \\
C_{e q}^{(0)} & =\mathcal{G}\left(\frac{\alpha_{C}}{\beta_{C}}, \frac{\alpha_{B}}{\beta_{B}}, \frac{\alpha_{T}}{\beta_{T}}, 1-g_{B B}, g_{C B}, g_{x y}\right) \\
B_{e q}^{(0)} & =\mathcal{G}\left(\frac{\alpha_{C}}{\beta_{C}}, \frac{\alpha_{B}}{\beta_{B}}, \frac{\alpha_{T}}{\beta_{T}}, g_{B C}, 1-g_{C C}, g_{x y}\right)
\end{aligned}
$$

where

$$
\mathcal{G}\left(x, y, g_{x}, g_{y}, g_{x y}\right)=\left[x\left(1+h_{C T} T_{e q}^{(0)}\right)\right]^{\frac{g_{x}}{g_{x y}}}+\left[y\left(1-h_{B T} T_{e q}^{(0)}\right)\right]^{\frac{g_{y}}{g_{x y}}}
$$

is the auxiliary function with the fixed argument $g_{x y} \equiv g_{C C}\left(g_{B B}-1\right)-g_{C B} g_{B C}+1$. The formula emphasizes the scaling dependence on the ratios of the proliferative/apoptotic rates $\alpha_{T} / \beta_{T}, \alpha_{B} / \beta_{B}, \alpha_{C} / \beta_{C}$ and the exponents $\left(1-g_{B B}\right) / g_{x y}, g_{C B} / g_{x y}, g_{B C} / g_{x y},\left(1-g_{C C}\right) / g_{x y}$. The equilibrium becomes, obviously, not tumor-free, as $\alpha_{T} \neq 0$. When the simplifying assumption $g_{T C}=0$ is relaxed, the reliability of the formula for $T_{e q}^{(0)}$ can be further improved in an iterative way by revisiting condition $R_{T}=0$ and using the initial approximation $C \simeq C_{e q}^{(0)}$. Owing to the above steps, the improved approximation for tumoral population number $T_{e q}^{(0,+)}$ reflects the impact of OCs as follows

$$
\begin{aligned}
T_{e q}^{(0,+)} & =T_{e q}^{(0)}\left[\frac{\left(C_{e q}^{(0)}\right)^{g_{T C}}}{1+\frac{\alpha_{J}}{\beta_{T}} C_{e q}^{(0)}\left(1-\frac{\kappa_{J}}{\beta_{J}}\right)}\right]^{\frac{1}{1-g_{T T}}}, \\
J_{e q}^{(0,+)} & =\frac{\alpha_{J}}{\beta_{J}} C_{e q}^{(0)} T_{e q}^{(0,+)},
\end{aligned}
$$

where the population number $J_{e q}^{(0,+)}$ is obtained from $R_{J}=0$. In addition, we see that the above improvements of Eq. (3) exhibit the scaling form with arguments $\alpha_{J} / \beta_{T}, \kappa_{J} / \beta_{J}$ and $\alpha_{J} / \beta_{J}$. The approximate equilibrium 
solution of this type implicates the possibility of exploiting the influence of environmental characteristics $C_{e q}^{(0)}$ to affect the population of tumoral cells described by the $T_{e q}^{(0,+)}$ and $J_{e q}^{(0,+)}$ variables.

The essence of the environmental concept can also be captured analytically by examining a short-time nonequilibrium picture of environmental influence, which is discussed below. The straightforward quantification of the endogenous $(e d g)$ tumor response to the environment can be quantified by the two-time population ratios defined by

$$
f_{Y}^{e d g}(t, t+\Delta t) \stackrel{\text { def }}{=} \frac{Y(t+\Delta t)}{Y(t)}, \quad Y(t) \in\{T(t), J(t)\}
$$

considered for both tumoral population variants $Y(t)$ and sufficiently small $\Delta t$. In subsection 2.4.1 is the above preliminary concept revisited [see Eq. [18] in further] and discussed for larger separation intervals, i.e. for $\Delta t \rightarrow$ $\left(t_{E}-t_{S}\right)$.

Deeper understanding of the linkages between the environmental populations $B, C$ and the responses of population $T$ can be obtained simply by using the truncated short time Taylor expansion

$$
f_{Y}^{e d g}(t, t+\Delta t)=1+\frac{\Delta t R_{Y}(t)}{Y(t)}+\frac{(\Delta t)^{2}}{2 Y(t)} \frac{d R_{Y}}{d t}+\mathcal{O}\left(\Delta t^{3}\right)
$$

As will become clear later on, sufficient evidence of environmental influence can be obtained by expanding $f_{Y}^{e d g}(t, t+\Delta t)$ into $\Delta t^{2}$ order at least.

The first order represented by $d T / d t=f_{T}^{e d g} \sim R_{T}(C, T, J) / T, d J / d t=f_{J}^{e d g} \sim R_{J}(C, T, J) / J$ confirms that $C$ influences $d T / d t$ and $d J / d t$ while the impact of the changes due to environmental variable $B$ remains hidden. By means of the straightforward differentiation, the coefficients $\sim \frac{d R_{T}}{d t}, \frac{d R_{J}}{d t}$ corresponding to $\Delta t^{2}$ order from Eq.(7) can be expressed

$$
\begin{aligned}
\frac{1}{T} \frac{d R_{T}}{d t} & =\alpha_{T} C^{g_{T C}-1} T^{g_{T T}-2}\left(g_{T C} T R_{C}+g_{T T} C R_{T}\right) \\
& -\alpha_{J}\left(R_{C}+\frac{C}{T} R_{T}\right)+\kappa_{J} \frac{R_{J}}{T} \\
\frac{1}{J} \frac{d R_{J}}{d t} & =\alpha_{J}\left(\frac{T}{J} R_{C}+\frac{C}{J} R_{T}\right)-\beta_{J} \frac{R_{J}}{J}
\end{aligned}
$$

As both the above right-hand sides include $R_{C} \equiv R_{C}(T, C, B)$, the respective rates depend not only on $C$ (as in the first order case) but on $B$ as well, which makes the causal relation between environmental $(C, B)$ and tumoral $(T, J)$ populations more explicit. The short-time causality between exogenous parameter-induced environmental changes and tumor subsystems that closely applies to the present work can be captured in an analogous way.

\subsection{Sensitivity analysis and inverse/optimization task of ODEs system}

The utmost ambition of our work is the proposal of a numerical procedure that can be viewed as a kind of global sensitivity analysis designed for specific ODEs. We identify the most sensitive parameters or their combinations 


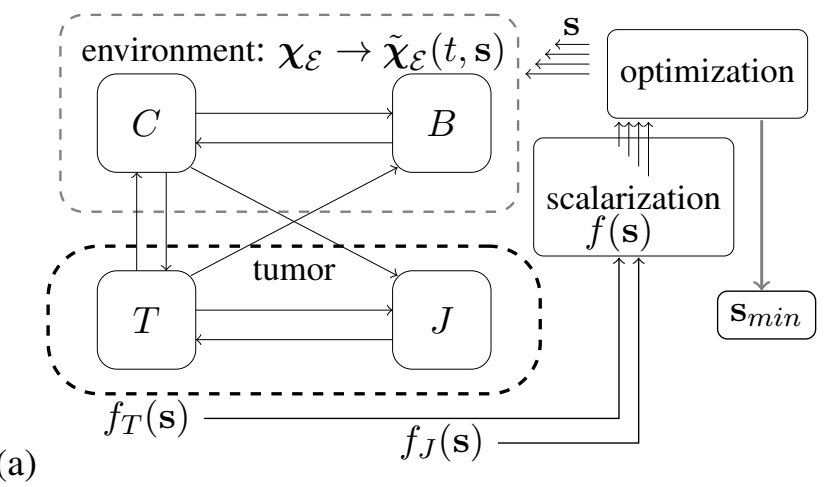

(b)

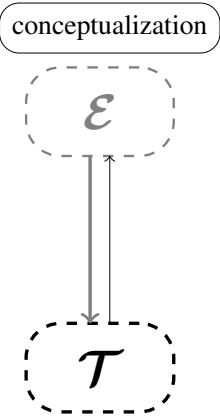

Figure 1: Schematic plot showing interactions (oriented graph edges) between the population numbers $C, B, T, J$, as described by Eqs.(1), (2). The topological representation of the algebraic structure of interactions and direct causal relations is presented in the part (a). As $R_{T}(C, T, J)$ does not include $B$, the directed link between nodes $B$ and $C$ absents. The links belong to the dependencies of the population rates [see Eqs.(1)]. The dynamics of environment is affected by the six pivotal parameters from Eq. (9) later replaced by smooth exogenous pulses [see Eqs.[10], [13] ]. The scheme also clarifies the influence of discrete $\mathbf{s}$ (string) parameters from Eq. (12) which determine the relations between $C, B$ (environment) and the tumoral populations $T$, $J$. The scheme is supplemented by the computational aspects related to the choice of the objective functions $f_{T}, f_{J}$, their scalarization (see subsection 2.4.1 and sec 4) and optimization leading to the optimal $\mathbf{s}_{\min }$ [see Eq.(20)]. In part (b) we depict the nodes corresponding to the conceptual framework from section 7 where the only pair of abstract population vectors $\mathcal{T}(t), \mathcal{E}(t)$ admits encompassing more general domain of population models with environments.

as potential intermediaries for an indirect virtual therapeutic intervention. For our purposes, which are mainly illustrative, the time-consuming search for the most influential and, hopefully, therapeutically promising parametric combinations is highly simplified. The parametric continuum is discretized and the search is conceived as the grid optimization. For a specified discretization of the parametric space, eventual inaccuracies may result from the numerical instabilities or stiffness of the respective ODE integrators [46].

As will be shown later, the optimality criteria are implicitly associated with the finite time interval, as the time horizon plays an important role in the decision-making. Moreover, to guarantee that the optimization selects for the solutions with controllable sparsity [47], the equality constraint or penalization term have been considered. The main concepts of the approach may be summarized as follows:

1. The analysis of indirect influence of the cellular environments represented by the dynamics of the population numbers $B(t)$ and $C(t)$ (or the respective rates $R_{B}, R_{C}$ ) on the tumoral populations quantified by $T(t), J(t)$. The scheme shown in Fig 1 outlines the topology of interactions and responses of $T, J$ to the exogenous parameters. Instead of analyzing intuitively obvious anti-proliferative/apoptotic effects 
controlled by the parameters $\alpha_{C}, \alpha_{B}, \beta_{C}, \beta_{B}$ we investigate sensitivity to the components of the tuple

$$
\chi_{\mathcal{E}}=\left[h_{C T}, g_{C C}, g_{C B}, h_{B T}, g_{B C}, g_{B B}\right],
$$

which parametrize $\left(1+h_{C T} T\right) C^{g_{C C}}, B^{g_{C B}}$ and $\left(1-h_{B T} T\right) C^{g_{B C}} B^{g_{B B}}$. (Here, the lower index $\mathcal{E}$ of $\chi_{\mathcal{E}}$ emphasizes the environmental dependence).

2. The dynamical instability generating tumor growth is induced by the superthreshold choice of the parameter $g_{T C}$ (see Table 1). (The parameter appears in the term $\sim C^{g_{T C}} T^{g_{T T}}$ which constitutes $R_{T}$.)

3. The constancy of the selected parameters listed in the tuple [Eq. (9)] is no longer considered, but the continuous in time dependencies will be introduced, where the original parameters are replaced by some exogenous time-varying functions [see Eq.(13) in the text below]

$$
\tilde{\chi}_{\mathcal{E}}(t, .)=\left[\widetilde{h}_{C T}(t, .), \widetilde{g}_{C C}(t, .), \widetilde{g}_{C B}(t, .), \widetilde{h}_{B T}(t, .), \widetilde{g}_{B C}(t, .), \widetilde{g}_{B B}(t, .)\right]
$$

purposefully constructed to stay in the vicinity of their respective former static values. The proposal of the specific time dependencies implies the need for additional (discrete) parameters that not only control the switchover between the base functions but undergo optimization as well. Consequently, the constant parameters involved in $R_{B}, R_{C}$ are replaced by the corresponding time dependencies, formally

$$
\begin{aligned}
& R_{C}\left(\ldots, h_{C T}, g_{C C}, g_{C B}\right) \longrightarrow \widetilde{R}_{C}\left(\ldots, \widetilde{h}_{C T}(t, .), \widetilde{g}_{C C}(t, .), \widetilde{g}_{C B}(t, .)\right), \\
& R_{B}\left(\ldots, h_{B T}, g_{B C}, g_{B B}\right) \longrightarrow \widetilde{R}_{B}\left(\ldots, \widetilde{h}_{B T}(t, .), \widetilde{g}_{B C}(t, .), \widetilde{g}_{B B}(t, .)\right) .
\end{aligned}
$$

4. The inverse formulation is suggested which assumes the construction of the objective function $f($.$) which$ quantifies the degree of undesired behavior of $T(t)$ and $J(t)$. It follows that the aim of the optimization of $f($.$) is the selection of Eq. [10$ which admits tumor suppression for given time interval.

\subsection{Discretization of the parametric space, exogenous symmetric pulses}

The sensitivity analysis is based on the optimization of the exogenous stimulation from Eqs. (10), (11) which must be specified in more detail. In the present form it includes combinations of the auxiliary discrete parameters forming the strings

$$
\mathbf{s} \equiv\left[s_{I}, s_{h_{C T}}, s_{g_{C C}}, s_{g_{C B}}, s_{h_{B T}}, s_{g_{B C}}, s_{g_{B B}}\right] .
$$

The grid optimization passes through the space of possibilities, where each possibility is encoded by the string s. Continuity in time is guaranteed since two input pulses of prescribed shapes $\Psi_{C}\left(t, s_{I}\right), \Psi_{B}(t)$ modify the 
original Eq. (9) in a multiplicative manner as it follows

$$
\begin{aligned}
\widetilde{h}_{C T}\left(t, s_{h_{C T}}, s_{I}, Q\right) & =h_{C T}\left(1+Q s_{h_{C T}} \Psi_{C}\left(t, s_{I}\right)\right), \\
\widetilde{g}_{C C}\left(t, s_{g_{C C}}, s_{I}, Q\right) & =g_{C C}\left(1+Q s_{g_{C C}} \Psi_{C}\left(t, s_{I}\right)\right), \\
\widetilde{g}_{C B}\left(t, s_{g_{C B}}, s_{I}, Q\right) & =g_{C B}\left(1+Q s_{g_{C B}} \Psi_{C}\left(t, s_{I}\right)\right), \\
\widetilde{h}_{B T}\left(t, s_{h_{B T}}, Q\right) & =h_{B T}\left(1+Q s_{h_{B T}} \Psi_{B}(t)\right), \\
\widetilde{g}_{B C}\left(t, s_{g_{B C}}, Q\right) & =g_{B C}\left(1+Q s_{g_{B C}} \Psi_{B}(t)\right), \\
\widetilde{g}_{B B}\left(t, s_{g_{B B}}, Q\right) & =g_{B B}\left(1+Q s_{g_{B B}} \Psi_{B}(t)\right) .
\end{aligned}
$$

The parameter $Q \geq 0$ may be interpreted as the strength of therapeutic action. In the modeling below, we focus on the possible shapes of multi-dimensional pulse and their responses which can be used to model reversible variations of individual parameters. For that purpose, the discrete argument $s_{I}$ of $\Psi_{C}\left(t, s_{I}\right)$ is introduced [see Eq.(15) and illustrating Fig.2 below] to control timing and width of the pulse. Regarding the focus of our study, continuous (constant) administration is excluded because of the requirement of dominating autonomous regimes in the late-time systemic response which is equivalent to the condition of the presence of a rest period that allows for the recovery from toxicity [48].

The space of pulse configurations is determined by $\mathbf{s}$ including the four-state component $s_{I} \in \Omega^{(4)} \stackrel{\text { def }}{=}$ $\{0,1,2,3\}$, and six three-state components $s_{\text {index }} \in \Omega^{(3)} \stackrel{\text { def }}{=}\{-1,0,1\}$, where index $\in\left\{h_{C T}, g_{C C}, g_{C B}, h_{B T}\right.$, $g_{B C}, g_{B B}$. Next we will study optimality of the strings

$$
\mathbf{s} \in \Omega \stackrel{\text { def }}{=} \Omega^{(4)} \times \underbrace{\Omega^{(3)} \times \Omega^{(3)} \times \ldots \times \Omega^{(3)}}_{6 \times},
$$

where the cardinality $\operatorname{card}(\Omega)=4 \times 3^{6}=2916$ possible states.

The functions $\Psi_{C}\left(t, s_{I}\right), \Psi_{B}(t)$ used to carry out the sensitivity testing are modeled by means of the elementary symmetric pulses

$$
\begin{aligned}
\Psi_{B}(t) & =\psi\left(t, t_{0}, \xi_{0}, \sigma_{0}\right) \\
\Psi_{C}\left(t, s_{I}\right) & =\delta_{s_{I}, 0} \psi\left(t, t_{0}, \xi_{0}, \sigma_{0}\right)+\delta_{s_{I}, 1} \psi\left(t, t_{0}, \xi_{1}, \sigma_{0}\right) \\
& +\delta_{s_{I}, 2} \psi\left(t, t_{1}, \xi_{0}, \sigma_{0}\right)+\delta_{s_{I}, 3} \psi\left(t, t_{1}, \xi_{1}, \sigma_{0}\right)
\end{aligned}
$$

where $\delta_{s_{I}, s}$ denotes the Kronecker symbol, which equals to 1 or 0 depending on the match or mismatch status of $s_{I}$ and one of the referential values from $\Omega^{(4)}$. Note that the pulse timing and shaping encoded by $s_{I} \in \Omega^{(4)}$ undergo optimization as well. Incorporation of the sequential pulses controlled by $s_{I}$ is inspired by [49].

We remind that the role of the parameter $s_{I}$ is crucial, since it determines the timing and shape of the pulses; $\Psi_{B}($.$) is not affected by this parameter and serves as referential. For all possible pairs of arguments \left(t_{0}, \xi_{0}\right)$; $\left(t_{0}, \xi_{1}\right) ;\left(t_{1}, \xi_{0}\right) ;\left(t_{1}, \xi_{1}\right)$ including the time-translation $t_{t r} \in\left\{t_{0}, t_{1}\right\}$ and time rescaling factors $\xi_{s c} \in\left\{\xi_{0}, \xi_{1}\right\}$ we define four child wavelets [see Eq.[15)]

$$
\psi\left(t, t_{t r}, \xi_{s c}, \sigma_{0}\right)=\frac{1}{\sqrt{\xi_{s c}}} \phi\left(\frac{t-t_{t r}}{\xi_{s c}}, \sigma_{0}\right)
$$


which exploit the continuous-time univariate Gaussian symmetric model

$$
\phi(t)=\phi_{S}\left(t^{\prime}, \sigma_{0}\right)=\exp \left[-\frac{1}{2}\left(\frac{t^{\prime}}{\sigma_{0}}\right)^{2}\right],
$$

where $\sigma_{0}$ is the pulse width $\left[\sigma_{0}<\left(t_{1}-t_{0}\right)\right]$.

More realistic (asymmetric) variant of the function $\phi$ is introduced and studied in sec. 6. To avoid the direct impact of the pulses on the boundaries, the times $t_{0}, t_{1}$ were chosen to guarantee the localization of the pulse peaks far enough from the boundary values $t_{S}$ and $t_{E}$, where $t_{E}>t_{1}>t_{0}>t_{S}$, i.e. we assume $\psi \sim 0$ for $t \in\left\{t_{S}, t_{E}\right\}$. The time span $t_{E}-t_{S}-2 \sigma_{0}$ corresponds, at the conceptual level, to what is called the "drug holidays" [50, 51, 48]. Unlike the superposition of Dirac pulses, which define the control function in the abovecited reference, we have the finite pulse width $\sigma_{0}$. Obviously, the symmetry of input signal does not imply of the population responses. In particular, the unstable tumor growth causes irreversible changes which, in principal, can not be compensated solely by the action of reversible exogenous factors selected here. To summarize this section, the auxiliary functions $\Psi_{B}($.$) and \Psi_{C}($.$) were introduced to construct the reversible exogenous pulses$ of dimension six [see Eqs.(13) and (14)].

\subsection{Objective function, constrained optimization of tumor response}

In this section we focus on the optimization of tumor responses to the parametric environmental factors with the aim to identify those factors which lead to required changes of the tumoral subpopulations during some interval of observation. In conformity with the optimization theory, the objectives of indirect parametric and environmental manipulations with tumoral populations are quantified by the objective function. The proposed formulation consists of two main components. At first, the optimization component enables us to discriminate between many alternative therapeutic strategies (and to select, regarding the model, the optimum solution). Secondly, the component of sensitivity analysis enables more subtle understanding of the relationships between exogenous inputs and output variables in a system and, in addition, it enables to study degree of sparsity and robustness [51] of the proposed solutions. To pursue the above aims in our specific case of the model of unstable tumor growth, several key measures of tumor response are incorporated into the objective function.

\subsubsection{The measures of tumor responses, objective functions}

Below we introduce the key measures that reflect tumor responses in our specific case of unstable tumor growth. The time interval for the observation of the systemic dynamics is $\left\langle t_{S}, t_{E}\right\rangle\left(t_{S}\right.$ stands for the time of start, $t_{E}$ for the end). Further, we assume that population variations within the interval $\left\langle t_{S}, t_{E}\right\rangle$ do not contribute directly to the objective functions values that are calculated purely from the population characteristics at the interval endpoints.

Manipulation with several tumor populations requires multi-objective formulation. As the optimality should be evaluated not only for $T$ but for $J$ as well, two different objective functions are introduced

$$
f_{T}\left(t_{S}, t_{E}, \mathbf{s}\right) \stackrel{\text { def }}{=} \frac{T\left(t_{E}, \mathbf{s}\right)}{T\left(t_{S}\right)}, \quad f_{J}\left(t_{S}, t_{E}, \mathbf{s}\right) \stackrel{\text { def }}{=} \frac{J\left(t_{E}, \mathbf{s}\right)}{J\left(t_{S}\right)},
$$


where the notation emphasizes the conditioning by discrete $\mathbf{s}$. We formulate the objective function in the terms of relative (instead of absolute) tumor abundances to be able to stabilize non-free tumor equilibria by environmental moves (e. g. the pulses of therapy). Such formulation of the objective function is in line with the concept of adaptive therapy [13]. The alternative formulation exists [29] based on the OBs and OCs abundances that aims to restore their balance disrupted by MM.

Productive and broadly accepted paradigm in the area of the multi-objective optimization is the Wald's "minimax" optimality [52, 53, 54]. The scalarization procedure was designed to solve multi-objective decision-making problems where the decisions are made on the basis of the worst possible choice. Owing to this, both measures from Eq.(18) can be incorporated into the single objective function form

$$
f\left(t_{S}, t_{E}, \mathbf{s}\right) \stackrel{\text { def }}{=} \max \left\{f_{T}\left(t_{S}, t_{E}, \mathbf{s}\right), f_{J}\left(t_{S}, t_{E}, \mathbf{s}\right)\right\}
$$

where the optimal string $\mathbf{s}=\mathbf{s}_{\min }$ is defined in the standard way

$$
\mathbf{s}_{\min } \stackrel{\text { def }}{=} \arg \min _{\mathbf{s} \in \Omega} f\left(t_{S}, t_{E}, \mathbf{s}\right) \text {. }
$$

At the moment, we do not incorporate metastatic potential and drug resistance consequent to the JCs [30, 36] into the model. In the future, an eventual metastatic population derived from the JCs could be incorporated as the third component of the objective function.

In order to control the number of non-zero s components $(+1$ corresponds to stimulation, while -1 stands for inhibition), the optimization task from Eq.20 is supplemented with the constraint

$$
\delta_{0, s_{h_{C T}}}+\delta_{0, s_{g_{C C}}}+\delta_{0, s_{g_{C B}}}+\delta_{0, s_{h_{B T}}}+\delta_{0, s_{g_{B C}}}+\delta_{0, s_{g_{B B}}}=n_{0}(\mathbf{s}),
$$

where $n_{0}(\mathbf{s})=0,1, \ldots, 6$ is the number of $\mathbf{s}$ components that are equal to $0 ; \delta_{s_{x}, s_{y}}$ is the Kronecker symbol.

The variable $s_{I} \in \Omega^{(4)}$ absents in the 1.h.s. of Eq. 21 as it relates only to the timing and width of the pulses (Eq.(15)).

As up-to-date anticancer therapies are accompanied by the variety of negative or uncertain side effects, the constraints may represent the elementary quantification of their impacts [48]. When $6-n_{0}$ increases, the tendency of the system towards more complex response becomes unavoidable. Therefore, because of direct link between the number of constraints and parametric sparsity, the term $1-\left(n_{0} / 6\right)$ may be interpreted as the index of parametric redundancy [55]. More compact, constraint-free reformulation where adverse effects are quantified by $1-\left(n_{0} / 6\right)$ regularization term is presented in sec. 5 .

\subsection{Evaluation of the model outputs}

The optimum value of the objective function

$$
f_{\min } \equiv f_{\min }\left(t_{S}, t_{E}\right) \stackrel{\text { def }}{=} \min _{\mathbf{s} \in \Omega} f\left(t_{S}, t_{E}, \mathbf{s}\right)=f\left(t_{S}, t_{E}, \mathbf{s}_{\min }\right)
$$


enables straightforward quantification of the quality of the optimization outputs. The disparities in the optimization of $f_{T}$ and $f_{J}$ are represented by the measure

$$
D f_{\min } \stackrel{\text { def }}{=}\left|\min _{\mathbf{s} \in \Omega} f_{T}-\min _{\mathbf{s} \in \Omega} f_{J}\right| .
$$

In addition, to understand how the choice of the fitness variant affects $\mathbf{s}_{\min }$, we introduced the mean Hamming distance

$$
d_{H, \text { smin }} \stackrel{\text { def }}{=} \frac{1}{7} \sum_{\{\forall \mathbf{s} \text { components }\}} \mathbb{1}\left(\arg \min _{s \in \Omega} f_{T} \neq \arg \min _{s \in \Omega} f_{J}\right),
$$

where $\mathbb{1}($.$) is the indicator function of the logical-type argument, which returns one when the argument is True$ and zero otherwise. Having defined a per-component distance measure, we consider the normalization factor $1 / 7$.

\section{Numerical implementation, symmetric pulses}

\subsection{Parameter settings}

The parameters used in all simulations are consistent with those used by Koenders and Saso [9]. Their values are listed in Table 1. Additional in our approach are the parameters which define the exogenous dynamics of the functions $\psi\left(t, t_{t r}, \xi_{s c}, \sigma_{0}\right), \phi_{S}\left(t, \sigma_{0}\right)$ [see Eq. [16] and Eq.[17]] which provide $\Psi_{B}(t), \Psi_{C}\left(t, s_{I}\right)$ [see Eq. [15)] for given $s_{I}$; their values are

$$
t_{0}=140 \text { day }>t_{S}=100 \text { day, } t_{0}<t_{1}=160 \text { day }<t_{E}=200 \text { day }
$$

and the scaling parameters needed for the evaluation of the functions

$$
\xi_{0}=1, \xi_{1}=0.5 \text { (scaling factors) }, \quad \sigma_{0}=7 \text { day (pulse width) } .
$$

The pulse width $2 \sigma_{0}=14$ day has been chosen to be roughly consistent with the half-life of MM cells that is $\sim 10-20$ days. The choice $t_{E}-t_{S}=100$ day was primarily motivated by elsewhere referred average time for the restoration of the population size towards equilibrium [8, 9]. The importance of this time scale is supported by the work [28].

The overall dynamics and system responses were obtained by means of the fourth-order Runge Kutta (RK4) method with the integration step $\delta t=5 \times 10^{-4}$ day $\sim 8.64 \mathrm{sec}$.

\subsubsection{Static equilibrium - stability}

Before going into the details of the parameter settings in dynamic approach, we briefly discuss some of the static results. The approximate population equilibrium $B=B_{e q}^{(0)}=230.87, C=C_{e q}^{(0)}=1.685, T=T_{e q}^{(0)}=9.0$ was calculated using Eqs. (3), (4), adopted from [9]. We note that the "zero-order" equilibrium did not take 


\begin{tabular}{|c|c|c|c|c|c|}
\hline param. val. & class & param. val. & class & param. val. & class \\
\hline$\alpha_{C}=3.0 d_{a y}-1$ & $R_{C}$ & $\alpha_{B}=4.0 d_{a y}-1$ & $R_{B}$ & $\alpha_{T}=0.3 d a y^{-1}$ & $R_{T}$ \\
\hline$\alpha_{J}=0.001 \mathrm{day}^{-1}$ & $R_{J}$ & $\beta_{C}=0.2 d a y^{-1}$ & $R_{C}$ & $\beta_{B}=0.02 d a y^{-1}$ & $R_{B}$ \\
\hline$\beta_{T}=0.1 d a y^{-1}$ & $R_{T}$ & $\beta_{J}=0.3 d a y^{-1}$ & $R_{J}$ & $\kappa_{J}=0.3 d a y^{-1}$ & $R_{T}$ \\
\hline$h_{B T}=0.035$ & $R_{B}$ & $h_{C T}=h_{B T}$ & $R_{C}$ & $g_{C C}=0.5$ & $R_{C}$ \\
\hline$\overline{g_{C B}}=-0.5$ & $R_{C}$ & $\overline{g_{B C}=1.0}$ & $R_{B}$ & $\overline{g_{B B}}=0.0$ & $R_{B}$ \\
\hline$\overline{g_{T T}=0.5}$ & $R_{T}$ & & & $\overline{g_{T C, \text { init }}=}=0.0$ & $R_{T}$ \\
\hline$\overline{g_{T C, s t a b}}=0.3$ & $R_{T}$ & $g_{T C, t h r}=0.3648$ & $R_{T}$ & $g_{T C, u n s t a b}=0.37$ & $R_{T}$ \\
\hline
\end{tabular}

Table 1: The constant model parameters used in all simulations. The parameter values (param. val.) are adopted from the work [9]. They are divided into four classes - four rates, $R_{C}, R_{B}, R_{T}, R_{J}$ according to the occurrence on the right hand side of Eq. 22). The underlined parameters $h_{B T}, \ldots, g_{T T}$ are later replaced by their corresponding time-varying analogs [see Eq. (13)]. Four variants of the parameter $g_{T C}$ (bottom rows of the table) were used to simulate the stability/instability of steady states in the tumor growth context.

into account population of the JCs $(J=0)$. In addition, the approximation assumed $g_{T C}=g_{T C \text {, init }}=0.0$. By using asymptotes of direct integration of Eqs.(1) and (2) for appropriately chosen nonzero constant value $g_{T C}=g_{T C, s t a b}=0.3$, the previous equilibrium estimates changed and the asymptotically stable equilibrium $B_{e q}^{(1)}=211.42, C_{e q}^{(1)}=2.052, T_{e q}^{(1)}=13.854, J_{e q}^{(1)}=0.0947$ was obtained.

\subsection{Initiation, imbalance, pathogenesis and MM progression}

Our modeling of initiation and promotion of MM pathogenesis takes into account early steps of OCs involvement in that process. According to [56], the direct interactions between MM cells and OCs may increase MM proliferation which is mediated by $\sim C^{g_{T C}} T^{g_{T T}}$ term. Therefore, we assume that the large pathogenic irreversible changes can be modeled with increased $g_{T C}$ which causes unstable growth of the populations of MM cells. The parametric shift in $g_{T C}$ exceeding $g_{T C, s t a b}$ results in the permanent instability that may be attributed to the abnormal cell signaling, modulation of microenvironment, or dysregulation that irreversibly degrades bone. From the point of view of the evolutionary game theory [26], the shift can be attributed to the perturbation of OC-MM coexistence line. A more microscopic interpretation can be found in [57, 29], where the MM growth is stimulated by OCs secretion of $\mathrm{TNF}_{\alpha}$ cytokine.

We have numerically found that the parameter $g_{T C}$ reveals the threshold value $g_{T C, t h r} \simeq 0.3648$ which separates the stable equilibrium from the unstable regime. Consequently, the supercritical value $g_{T C, u n s t a b}=$ $0.37>g_{T C, t h r}$ was used in the tumor growth simulations. The unstable system is integrated over the initial $t_{S}=100$ day with constant parameters, i. e. for $Q=0$. This period is used to simulate the uncontrolled growth. The resulting values $T\left(t_{S}\right)=16.784$ and $J\left(t_{S}\right)=0.132$ have been stored for later comparative purposes. After this period, the tumor suppression is purposefully initialized. Subsequently, the equations were integrated for the time interval $\left\langle t_{S}, t_{E}\right\rangle$, where $t_{E}=200$ day. The pairs $\left(T\left(t_{S}\right), T\left(t_{E}\right)\right),\left(J\left(t_{S}\right), J\left(t_{E}\right)\right)$ were used to calculate the objective function defined by Eq.(18). In the case $Q=0$ (without pulses), when there is 
no dependence on s (total degeneracy) and, consequently, no optimization is possible, the calculation provides $f=f_{J}=1.1568>f_{T}=1.0681$. This result suggests that, at least in the low $Q$ region, more rapidly expanding population of the JCs could be suppressed with higher priority [in the light of the criterion specified in Eq. (19).

\subsection{Results, symmetric pulses}

Before presenting the simulation results, we visualize examples of the elementary Gaussian exogenous pulses [see Eq.(15), Fig 2] for different $s_{I}$. Next, instead of immediate quest for the optimality, we find instructive to inspect the entire search space. Our findings (see Fig. 3) demonstrate that not all the strings $\mathbf{s} \in \Omega$ are tumor suppressive, i. e. providing $f()<$.1 . It seems that the outputs quantified by $f($.$) split into qualitatively$ different branches. One may identify the options $\mathbf{s} \in \Omega$ corresponding to the quasi-equilibrium (line $f=1$ ), unstable tumor growth $(f>1)$, or temporary tumor suppression $(f<1)$, respectively. It can be seen that for too small $Q$ the desired effects $(f<1)$ can not be achieved, and growth instability may be suppressed only for $Q>Q_{t h r}=0.049$. It demonstrates that the parametrization covers a broad range of scenarios, so that optimization can affect the therapeutic efficiency.

After illustrating all the possible scenarios contained in $\Omega$, we turn to the analysis of the optimal selections (see Fig 4). It seems that the small and large $Q$ regions differ in qualitative as well as quantitative components. The complex form of $f_{\text {min }}(Q)$ consisting of several sharp folds arises at large $Q$. This is because of combined effect of discretization, selection, and nonlinearities, as well as the strength of exogenous factors. With increasing $n_{0}$, the sharp irregular folds in the $f_{\min }(Q)$ dependencies become smoother (see Fig 4). Such lowered sensitivity to $Q$ arises due to very restrictive constraints (constructed for high $n_{0}$ ) corresponding to the limited number of parameters with lowered sensitivity to $Q$. In Fig 5 we see how the invasiveness of the MM and JCs varies with $Q$. The characteristics $D f_{\min }(Q)$ shows $\mathrm{V}$-shaped $\left(n_{0}\right.$-dependent) minima that reflect the compensatory effect of $f_{T}$ and $f_{J}$ localized slightly above the threshold values $Q_{t h r}\left(n_{0}\right)$.

For further details, we refer the interested reader to Appendix, which contains discussion of the periodic extensions of the solutions obtained as well as the stability of the RK4 method and its comparison with the implicit integration methods, regarding specific aspects of our application.

The results of constrained optimization with $n_{0}=0$ are presented in Tab. 2. Similarly, the systematic analysis covers $n_{0}=1$ (see Tab. 3), $n_{0}=2$ (Tab. 4), $n_{0}=3$ (Tab. 5), $n_{0}=4$ (Tab. 6). This demonstrates that the optimized components of $\mathbf{s}_{\text {min }}$ are interdependent. In fact, these outputs, apart from underlying complexity of the responses, answer the questions about the parametric sensitivity in the particular regions of $\left(Q, n_{0}\right)$ plane. Apparently, the components of $\mathbf{s}_{\min }$ differ not only in the persistence of the prevailing discrete values, but in the tendencies to make jumps along the $Q$ coordinate as well. The complexity of the $\mathbf{s}_{\min }\left(Q, n_{0}\right)$ emerges from the nonlinearity which destabilizes the form of exogenous pulses. As the available options for the exogenous influence narrow down with $n_{0}$, the relative influence of certain parameters becomes more apparent.

Although a detailed interpretation of the structure of $\mathbf{s}_{\min }$ strings may be difficult, a few instructive facts outline their implicit logic. Some of the results are relatively robust to the specific assumptions, including the choice of $Q$ or $n_{0}$. To illustrate this, we reconsider the solution $\mathbf{s}_{\min }=[3,-1,-1,+1,-1,+1,-1]$ obtained for $n_{0}=0, Q \in\langle 0.01,0.27\rangle$ (Tab. 2. Tab. 3). This string shows essential overlap with that of the neighboring interval $Q \in\langle 0.27,0.63\rangle$. The only difference is in the sensitivity of the third component $\left(s_{g_{C C}}=+1\right) \rightarrow$ 
$\left(s_{g_{C C}}=-1\right)$. Interestingly, the same component destabilizes $\left[\left(s_{g_{C C}}=-1\right) \rightarrow\left(s_{g_{C C}}=0\right)\right]$ when the constraint $n_{0}=1$ is applied. In addition, we see that the therapy applied to the both feedback components, $s_{h_{C T}}=-1$ and $s_{h_{B T}}=-1$, acts against the tumor influence on OCs and OBs. This is in line with autocrine inhibition of OCs by $s_{g_{C C}}=-1$ which may reduce replication rate of the tumoral cells $\sim \alpha_{T} C^{g_{T C}} T^{g_{T T}}$. More universal perspective can be captured from the comparison in Tabs. 22.5. Here, the highest stability is achieved for $s_{h_{B T}}=-1$ and $s_{g_{B C}}=+1$, while $s_{g_{C B}}$ is very sensitive to $Q$. However, as shown in Tabs. 6.7, the role of $s_{g_{C B}}$ is unique, as its nonzero values are robust against selection imposed by the constraints with $n_{0} \geq 4$. In this case, the zero values of $s_{h_{C T}}, s_{g_{C C}} s_{h_{B T}}, s_{g_{B B}}$ are quite persistent with respect to $Q$. Within the resulting optimal sparse solution, the effect of therapeutically relevant timing is controlled by $s_{I}$ coupled with $s_{g_{C B}}=+1$ and $s_{g_{B C}}=-1$.

\begin{tabular}{|r|c||c|cccccc|}
\hline $\begin{array}{c}\text { inter. length } \\
\times 100\end{array}$ & $\begin{array}{c}Q \\
\text { interval }\end{array}$ & $s_{I}$ & $s_{h_{C T}}$ & $s_{g_{C C}}$ & $s_{g_{C B}}$ & $s_{h_{B T}}$ & $s_{g_{B C}}$ & $s_{g_{B B}}$ \\
\hline 26 & $<0.01,0.27>$ & 3 & - & - & + & - & + & - \\
36 & $<0.27,0.63>$ & 3 & - & + & + & - & + & - \\
12 & $<0.63,0.75>$ & 0 & - & + & - & - & + & - \\
8 & $<0.75,0.83>$ & 1 & - & + & - & - & + & - \\
42 & $<0.83,1.25>$ & 0 & + & - & - & - & + & - \\
3 & $<1.25,1.28>$ & 1 & + & - & - & - & + & - \\
58 & $<1.28,1.86>$ & 0 & - & - & - & - & + & - \\
13 & $<1.86,1.99>$ & 1 & - & - & - & - & + & - \\
30 & $<1.99,2.29>$ & 3 & - & + & + & - & + & - \\
102 & $<2.29,3.31>$ & 1 & - & + & + & - & - & - \\
168 & $<3.31,4.99>$ & 1 & - & - & + & - & - & - \\
\hline
\end{tabular}

Table 2: The results of constrained optimization with $n_{0}=0$. The relations between $Q$ and $\mathbf{s}_{\min }$ remain persistent for given intervals of $Q$ (i.e. for the respective line of the table). The lengths of the $Q$ intervals are given in the first column. The columns $s_{I}, s_{h_{C T}}, \ldots, s_{g_{B B}}$ correspond to the optimum $\mathbf{s}_{\min }$. The notation $-\equiv-1,+\equiv 1$ is used here as well as for all the tables with the similar structure.

\section{Alternative scalarizations: linkage between the worst-case and the best-case}

In this section we address the particular issue how the optimization output depends on the particular scalarization of the original multi-objective problem considering the objectives $f_{T}$ and $f_{J}$, which further opens the question of the stability of the optima. For a stable scalarization, the small variation in the original formula results in the small change in the optimum obtained. To investigate the issue of eventual alternatives to the benchmark scalarization by the worst-case scenario, we propose the following framework requirements: (a) the alternative scalarization is parametrized by the single real parameter which quantifies deviation from the worst-case limit; (b) the invariance under the exchange of $f_{T}$ and $f_{J}$; (c) scalarizations proceed from the generalized mean that 


\begin{tabular}{|r|c||c|cccccc|}
\hline $\begin{array}{c}\text { inter. length } \\
\times 100\end{array}$ & $\begin{array}{c}Q \\
\text { interval }\end{array}$ & $s_{I}$ & $s_{h_{C T}}$ & $s_{g_{C C}}$ & $s_{g_{C B}}$ & $s_{h_{B T}}$ & $s_{g_{B C}}$ & $s_{g_{B B}}$ \\
\hline 25 & $<0.01,0.26>$ & 3 & - & - & + & - & + & 0 \\
$<1$ & $<0.27,0.27>$ & 3 & - & 0 & + & - & + & - \\
35 & $<0.28,0.63>$ & 3 & - & + & + & - & + & 0 \\
6 & $<0.64,0.70>$ & 0 & - & + & - & - & + & 0 \\
14 & $<0.71,0.85>$ & 0 & + & 0 & - & - & + & - \\
20 & $<0.86,1.06>$ & 0 & - & 0 & - & - & + & - \\
8 & $<1.07,1.15>$ & 0 & + & - & - & - & + & 0 \\
18 & $<1.16,1.34>$ & 0 & 0 & - & - & - & + & - \\
28 & $<1.35,1.63>$ & 0 & - & - & - & - & + & 0 \\
92 & $<1.64,2.56>$ & 0 & + & + & 0 & - & + & - \\
44 & $<2.57,3.01>$ & 1 & + & + & 0 & - & + & - \\
80 & $<3.02,3.82>$ & 1 & - & 0 & + & - & - & - \\
19 & $<3.83,4.02>$ & 1 & - & - & + & - & - & 0 \\
$<1$ & $<4.03,4.03>$ & 1 & - & - & 0 & - & - & - \\
95 & $<4.04,4.99>$ & 1 & - & - & + & - & - & 0 \\
\hline
\end{tabular}

Table 3: The results of the $f\left(t_{S}, t_{E}, \mathbf{s}\right)$ minimization constrained by $n_{0}=1$. The differences in $\mathbf{s}_{\min }$ obtained for different intervals of $Q$. Total persistence of the $s_{h_{B T}}=-1$. Relatively high persistence of $s_{g_{B C}}=1$ for $Q<3$ (See results of synergistic analysis in sec 6.2 below). Highest number of zeros and highest frequency of the changes between -1 and 0 is shown in the column $s_{g_{B B}}$.

expresses the central tendencies of $f_{T}$ and $f_{J}$. The alternative which fulfills the above requirements is Lehmer mean [42]

$$
f_{L}\left(p_{L}\right)=\frac{f_{T}^{p_{L}}+f_{J}^{p_{L}}}{f_{T}^{p_{L}-1}+f_{J}^{p_{L}-1}}
$$

which we use in the further analysis. The family of scalarized variants $\left\{f_{L}\left(p_{L}\right) \mid p_{L} \in \mathbb{R}\right\}$ uses $p_{L}$ to bridge the worst-case $f_{L}\left(p_{L} \rightarrow \infty\right)=\max \left\{f_{T}, f_{J}\right\}$ and the best-case $f_{L}\left(p_{L} \rightarrow-\infty\right)=\min \left\{f_{T}, f_{J}\right\}$ (therapeutically infeasible) limits. To quantify the level of stability, the global optimum $\mathbf{s}_{L, \min }=\arg \min _{s \in \Omega} f_{L}\left(p_{L}\right)$ is compared with the optimum corresponding to the worst-case limit via the alternative mean Hamming distance

$$
d_{L, \text { smin }}\left(p_{L}\right)=\frac{1}{7} \sum_{\{\forall \mathbf{s} \text { components }\}} \mathbb{1}\left(\arg \min _{s \in \Omega} f_{L}\left(p_{L}\right) \neq \arg \min _{s \in \Omega} f\right) .
$$

constructed in complete analogy to Eq. 24). The results of the numerical analysis of $d_{L, \text { smin }}$ corresponding to the case $n_{0}=0$ (other constraints show an analogous behavior) are presented in Fig 6 . They confirm qualitative differences between negative (unstable, i.e. considerably different from the worst-case), and stable domains of sufficiently large $p_{L}>0$. The additional information obtained from the calculations is that scalarizations 


\begin{tabular}{|r|c|c|cccccc|}
\hline $\begin{array}{c}\text { inter. length } \\
\times 100\end{array}$ & $\begin{array}{c}Q \\
\text { interval }\end{array}$ & $s_{I}$ & $s_{h_{C T}}$ & $s_{g_{C C}}$ & $s_{g_{C B}}$ & $s_{h_{B T}}$ & $s_{g_{B C}}$ & $s_{g_{B B}}$ \\
\hline 4 & $<0.01,0.05>$ & 3 & 0 & - & + & - & + & 0 \\
42 & $<0.06,0.48>$ & 3 & - & 0 & + & - & + & 0 \\
13 & $<0.49,0.62>$ & 3 & 0 & + & + & - & + & 0 \\
15 & $<0.63,0.78>$ & 0 & + & 0 & - & - & + & 0 \\
10 & $<0.79,0.89>$ & 0 & 0 & 0 & - & - & + & - \\
17 & $<0.90,1.07>$ & 0 & - & 0 & - & - & + & 0 \\
2 & $<1.08,1.10>$ & 1 & - & 0 & - & - & + & 0 \\
23 & $<1.11,1.34>$ & 0 & 0 & - & - & - & + & 0 \\
12 & $<1.35,1.47>$ & 1 & 0 & - & - & - & + & 0 \\
108 & $<1.48,2.56>$ & 0 & + & + & 0 & - & + & 0 \\
44 & $<2.57,3.01>$ & 1 & + & + & 0 & - & + & 0 \\
100 & $<3.02,4.02>$ & 1 & - & 0 & + & - & - & 0 \\
0 & $<4.03,4.03>$ & 1 & - & - & 0 & - & - & 0 \\
95 & $<4.04,4.99>$ & 1 & - & 0 & + & - & - & 0 \\
\hline
\end{tabular}

Table 4: The optimized components of $\mathbf{s}_{\min }$ (see Eq.(20)) obtained for the constraint $n_{0}=2$ (see Eq.(21)).

performed for the small $Q$ region (approximately $Q \lesssim 0.2$ ) are relatively well stabilized. This contrasts with the optimal $\mathbf{s}_{L, \min }$ corresponding to the higher $Q$ values, where sensitivity to $p_{L}$ becomes pronounced. We see that computational (and, eventually, therapeutic) problems lie mainly in the domain of high $Q$ values, where the irregular and less persistent behavior of $d_{L, s m i n}\left(p_{L}\right)$ can be observed.

\section{Modified objective function with the penalty term, sparse optimization}

We completed our work by experimenting with the alternative worst-case scalarized objective function

$$
f\left(t_{S}, t_{E}, \mathbf{s}, \lambda\right)=\max \left\{f_{T}\left(t_{S}, t_{E}, \mathbf{s}\right), f_{J}\left(t_{S}, t_{E}, \mathbf{s}\right)\right\}+\lambda Q\left(1-\frac{n_{0}(\mathbf{s})}{6}\right),
$$

supplemented by the extra penalty term $\lambda Q\left(1-n_{0}(\mathbf{s}) / 6\right)$ aimed at the selection of representative parameters [47]. The side effects of the therapy are proportional to the regularization parameter $\lambda \geq 0$ and previously introduced strength of the therapeutic action $Q$. The factor $\left(1-n_{0}(\mathbf{s}) / 6\right) \in(0,1)$ accounts for the fraction of nonzero s components. To sum up, comparing it to the function $f\left(t_{S}, t_{E}, \mathbf{s}\right)$ Eq. 19 , the above formulation minimizes unpredictable toxic side effects of the therapy [48] by preferring weaker interventions. Moreover, strict constraint on $\mathbf{s}$ no longer applies. In addition, we note that the sparsity-inducing penalization introduced by Eq. 29] is analogous to that adopted when considering the effective Markowitz portfolio diversification [58].

In Fig 7 we present numerical results obtained for the function Eq. 29]. They reveal that the optimum of $f\left(t_{S}, t_{E}, \mathbf{s}, \lambda\right)$ exists not only in $\mathbf{s}$, but along $Q$ for large enough $\lambda(\lambda>0.3)$ as well. Highly inefficient effects 


\begin{tabular}{|r|c|c|cccccc|}
\hline $\begin{array}{c}\text { inter. length } \\
\times 100\end{array}$ & $\begin{array}{c}Q \\
\text { interval }\end{array}$ & $s_{I}$ & $s_{h_{C T}}$ & $s_{g_{C C}}$ & $s_{g_{C B}}$ & $s_{h_{B T}}$ & $s_{g_{B C}}$ & $s_{g_{B B}}$ \\
\hline$<1$ & $<0.01,0.01>$ & 3 & 0 & - & + & - & 0 & 0 \\
59 & $<0.02,0.61>$ & 3 & 0 & 0 & + & - & + & 0 \\
31 & $<0.62,0.93>$ & 0 & 0 & 0 & - & - & + & 0 \\
25 & $<0.94,1.19>$ & 1 & 0 & 0 & - & - & + & 0 \\
26 & $<1.20,1.46>$ & 3 & 0 & 0 & + & - & + & 0 \\
141 & $<1.47,2.88>$ & 0 & 0 & + & 0 & - & + & 0 \\
210 & $<2.89,4.99>$ & 1 & 0 & 0 & + & - & - & 0 \\
\hline
\end{tabular}

Table 5: The effect of the constraint $n_{0}=3$.

\begin{tabular}{|r|c||c|cccccc|}
\hline $\begin{array}{c}\text { inter. length } \\
\times 100\end{array}$ & $\begin{array}{c}Q \\
\text { interval }\end{array}$ & $s_{I}$ & $s_{h_{C T}}$ & $s_{g_{C C}}$ & $s_{g_{C B}}$ & $s_{h_{B T}}$ & $s_{g_{B C}}$ & $s_{g_{B B}}$ \\
\hline 169 & $<0.01,1.70>$ & 3 & 0 & 0 & + & - & 0 & 0 \\
35 & $<1.71,2.06>$ & 0 & 0 & + & 0 & 0 & + & 0 \\
44 & $<2.07,2.52>$ & 1 & 0 & 0 & + & 0 & - & 0 \\
247 & $<2.53,4.99>$ & 1 & 0 & 0 & + & 0 & - & 0 \\
\hline
\end{tabular}

Table 6: The optimized $\mathbf{s}_{\min }$ obtained for $n_{0}=4$. Compared to the low $n_{0}$, longer intervals in $Q$ occur.

correspond to $Q \gtrsim 0.8$. As expected [Fig 7(b)], in all the studied cases increase in $Q$ reduces the number of non-zero elements proportional to $1-(1 / 6)\left(n_{0}\right)_{\min }$ where $\left(n_{0}\right)_{\min }=n_{0}\left(\mathbf{s}_{\min }\right)$.

\section{The case of asymmetric pulses}

The symmetric pulses [see Eq. [17p] have been originally designed as a tool of the sensitivity analysis with unclear relationship to pharmacological applications. Because the pharmacologic characteristics of the pulse protocols [59] exhibit, in general, non symmetric, right tailed shape in time, we introduce more realistic asymmetric model in this section. By focusing on the exogenous changes, the model is introduced in two main steps. The first is

\begin{tabular}{|c|c||c|cccccc|}
\hline $\begin{array}{c}\text { inter. length } \\
\times 100\end{array}$ & $\begin{array}{c}Q \\
\text { interval }\end{array}$ & $s_{I}$ & $s_{h_{C T}}$ & $s_{g_{C C}}$ & $s_{g_{C B}}$ & $s_{h_{B T}}$ & $s_{g_{B C}}$ & $s_{g_{B B}}$ \\
\hline 498 & $<0.01,4.99>$ & 3 & 0 & 0 & + & 0 & 0 & 0 \\
\hline
\end{tabular}

Table 7: The optimization result for $n_{0}=5$ emphasizing the key role of $s_{g_{C B}}$. 
represented by the auxiliary formula

$$
\phi_{a}\left(t^{\prime}, \sigma_{a}, W_{a}, \tau_{a}\right)=\exp \left[-\frac{\left(t^{\prime}\right)^{2}}{2 \sigma_{a}^{2}}\left(1-\operatorname{logist}\left(\frac{t^{\prime}}{W_{a}}\right)\right)-\frac{t^{\prime}}{\tau_{a}} \operatorname{logist}\left(\frac{t^{\prime}}{W_{a}}\right)\right]
$$

incorporating the logistic function $\operatorname{logist}(\zeta)=1 /(1+\exp (-\zeta)) \in(0,1)$ of the dimensionless argument $\zeta=$ $t^{\prime} / W_{a}$, rescaling the time argument by the transition interval width $W_{a}$. The logistic local in time weighting causes that the characteristic time scale of the infusion/absorption regime $1 / \sigma_{a}$ is smoothly changing (on the time scale $1 / W_{a}$ ) to the late-time exponential drug elimination/excretion (depending on the metabolic conditions) with the characteristic time $\tau_{a}$.

The second step of the pulse definition reflects the fact that the impact of the drug depends not only on its concentration but on the effectiveness of the binding on the respective receptor as well, and it may be described by the transformation

$$
\phi_{A}\left(t^{\prime}\right) \equiv \phi_{A}\left(t^{\prime}, k_{E}, \sigma_{a}, W_{a}, \tau_{a}\right)=n_{E} \frac{\phi_{a}\left(t^{\prime}, \sigma_{a}, W_{a}, \tau_{a}\right)}{k_{E}+\phi_{a}\left(t^{\prime}, \sigma_{a}, W_{a}, \tau_{a}\right)}
$$

corresponding to the sigmoid model [59]. The form Eq.(31) replaces Eq.[17]; $n_{E}$ is the normalization parameter derived from the condition $\int_{-\infty}^{\infty} \mathrm{d} t \phi_{S}\left(t, \sigma_{0}\right)=\int_{-\infty}^{\infty} \mathrm{d} t \phi_{A}(t,$.$) . The normalization is proposed to achieve$ equivalence between the Gaussian $\left(\sigma_{0}=7 d a y\right)$ and the asymmetric pulses defined by $k_{E}, \sigma_{a}, W_{a}, \tau_{a}$. In the normalized case shorter duration is accompanied with enhanced intensity and vice versa. Two variants of the parameter $k_{E} \in\{0.1,1\}$ controlling the drug efficiency have been used in the numerical calculations: (a) $k_{E}=1$ corresponding to the high efficiency regime with normalization prefactor $n_{E}=1.46355$; (b) $k_{E}=0.1$ as a model of low drug efficiency with $n_{E}=0.44288$. In Fig 8 , the shape of asymmetric pulses is calculated for the numerical parameters $W_{a}=2 d a y, \sigma_{a}=3 d a y, \tau_{a}=14 d a y$ that we used in further optimizations.

The detailed comparison of the optimization results obtained for the symmetric and asymmetric pulses for the respective penalty functions [Eq. [29]] with the regularization parameters $\lambda \in\{0,0.2,0.4,0.6,0.8,1\}$ is presented in Tab. 8. In general, we conclude that (a) the pulse shape affects the optimization result, nevertheless the correlation of the optimal strings corresponding to the symmetric and asymmetric pulses is positive; (b) the values of $s_{I}$ encoding the pulse width are more susceptible to the pulse symmetry/asymmetry and drug efficiency $\left(k_{E}\right)$; (c) the nonzero value of $s_{g_{B B}}$ may disrupt the optimization results; (d) the value of $s_{g_{B C}}$ is

\begin{tabular}{|c|c|c|c|c|c|c|c|c|c|c|c|}
\hline (A) & \multicolumn{11}{|c|}{$\lambda=0$} \\
\hline Pulse & $k_{E}$ & $Q$ & $f_{\min }(\lambda)$ & $s_{I}$ & $s_{h_{C T}}$ & $s_{g_{C C}}$ & $s_{g_{C B}}$ & $s_{h_{B T}}$ & $s_{g_{B C}}$ & $s_{g_{B B}}$ & $d_{H, A-G}$ \\
\hline $\begin{array}{l}\text { asym.L } \\
\text { asym.H } \\
\text { Gauss }\end{array}$ & $\begin{array}{l}0.1 \\
1.0\end{array}$ & $\begin{array}{l}0.2 \\
0.2 \\
0.2\end{array}$ & $\begin{array}{l}0.7898 \\
0.7792 \\
0.8200\end{array}$ & $\begin{array}{l}2 * \\
2 *\end{array}$ & $=$ & $=$ & $\begin{array}{l}+90 B \\
+ \\
+\end{array}$ & $=$ & & $=$ & $\begin{array}{l}1 \\
1\end{array}$ \\
\hline $\begin{array}{l}\text { Gauss } \\
\text { asym.L } \\
\text { asym.H }\end{array}$ & $\begin{array}{l}0.1 \\
1.0\end{array}$ & $\begin{array}{l}0.2 \\
0.4 \\
0.4\end{array}$ & $\begin{array}{l}0.8200 \\
0.6246 \\
0.6105\end{array}$ & $\begin{array}{l}3 \\
2 * \\
2 *\end{array}$ & $=$ & $\begin{array}{l}\bar{t}^{*} \\
+ \\
+\end{array}$ & $\begin{array}{l}+ \\
+\end{array}$ & $\overline{-}$ & $\begin{array}{l}+ \\
+ \\
+\end{array}$ & $\overline{-}$ & $\begin{array}{l}2(1) \\
1\end{array}$ \\
\hline Gauss & 0 & 0.4 & $\frac{0.6667}{0.4892}$ & $\frac{3}{2 *}$ & $\overline{-}$ & $\stackrel{+}{+}$ & + & $\overline{-}$ & + & - & 1 \\
\hline $\begin{array}{l}\text { a } \\
\text { asym..H }\end{array}$ & 1.0 & 0.6 & 0.4637 & $2 *$ & - & + & + & - & $\begin{array}{r}+ \\
+\end{array}$ & $\overline{-}$ & 1 \\
\hline
\end{tabular}
relatively persistent for different $\lambda$ which coincides with its integral synergistic effect identified in sec.6.2. 


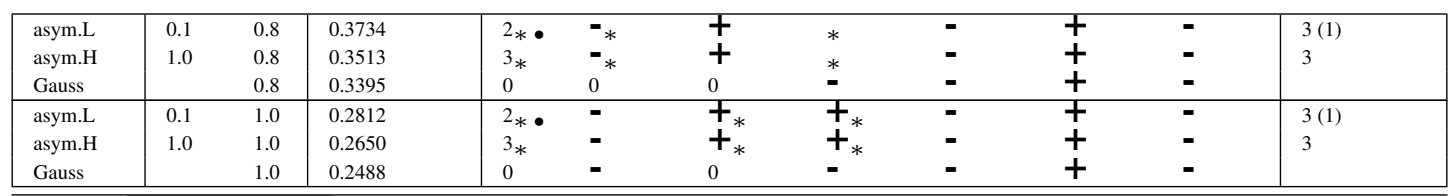

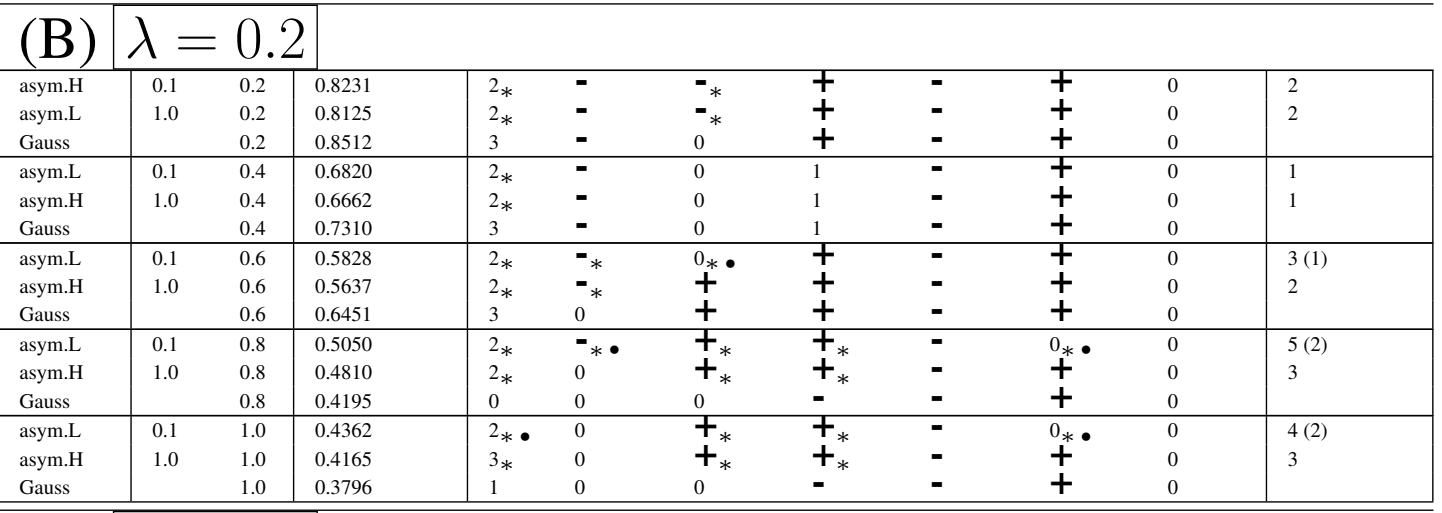

(C) $\lambda=0.4$

\begin{tabular}{|c|c|c|c|c|c|c|c|c|c|c|c|}
\hline $\begin{array}{l}\text { asym.L } \\
\text { asym.H } \\
\text { Gauss }\end{array}$ & $\begin{array}{l}0.1 \\
1.0\end{array}$ & $\begin{array}{l}0.2 \\
0.2 \\
0.2\end{array}$ & $\begin{array}{l}0.8560 \\
0.8434 \\
0.8778\end{array}$ & $\begin{array}{l}2 * \\
2 * \\
3\end{array}$ & $\begin{array}{l}\bar{z} \\
\overline{-}\end{array}$ & $\begin{array}{l}\overline{-}_{* \bullet} \bullet \\
0 \\
0\end{array}$ & $\begin{array}{l}+ \\
+ \\
+\end{array}$ & $\begin{array}{l}\bar{z} \\
\overline{-}\end{array}$ & $\begin{array}{l}0_{*} \bullet \\
+ \\
+\end{array}$ & $\begin{array}{l}0 \\
0 \\
0\end{array}$ & $\begin{array}{l}3(2) \\
1\end{array}$ \\
\hline asym.L & 0.1 & 0.4 & 0.7273 & $2 *$ & $\bar{I}_{*}$ & 0 & 7 & - & $0_{*} \bullet$ & 0 & $3(2)$ \\
\hline Gauss & & 0.4 & 0.7744 & 3 & 0 & 0 & + & - & + & 0 & \\
\hline asym.L & 0.1 & 0.6 & 0.6334 & $2 *$ & 0 & 0 & + & $=$ & $0_{*}$ & 0 & 2 \\
\hline asym.H & 1.0 & 0.6 & 0.6261 & $2 *$ & 0 & 0 & + & - & $0_{*}$ & 0 & 2 \\
\hline asym.H & 1.0 & 0.8 & 0.5612 & $3 *$ & 0 & 0 & + & - & $0_{*}$ & 0 & 3 \\
\hline Gauss & & 0.8 & 0.4995 & 0 & 0 & 0 & - & - & + & 0 & \\
\hline asym.L & 0.1 & 1.0 & 0.5017 & $2 * \bullet$ & 0 & 0 & & $0_{*}$ & $0_{*}$ & 0 & $4(1)$ \\
\hline asym.H & 1.0 & 1.0 & 0.5144 & $3 *$ & 0 & 0 & + & $0_{*}$ & $0_{*}$ & 0 & 4 \\
\hline Gauss & & 1.0 & 0.4796 & 1 & 0 & 0 & - & $=$ & + & 0 & \\
\hline
\end{tabular}

\begin{tabular}{|c|c|c|c|c|c|c|c|c|c|c|c|}
\hline Pulse & $k_{E}$ & $Q$ & $f_{\min }(\lambda)$ & $s_{I}$ & $s_{h_{C T}}$ & $s_{g_{C C}}$ & $s_{g_{C B}}$ & $s_{h_{B T}}$ & $s_{g_{B C}}$ & $s_{g_{B B}}$ & $d_{H, A-G}$ \\
\hline asym.L & 0.1 & 0.2 & 0.8776 & $2 *$ & $\bar{z}_{*}$ & 0 & 7 & - & $0_{*}$ & 0 & 3 \\
\hline asym.H & 1.0 & 0.2 & 0.8669 & $2 *$ & $\boldsymbol{-}_{*}$ & 0 & + & - & $0_{*}$ & 0 & 3 \\
\hline Gauss & & 0.2 & 0.8996 & 3 & 0 & 0 & + & - & + & 0 & \\
\hline asym.L & 0.1 & 0.4 & 0.7560 & $2 *$ & 0 & 0 & + & - & 0 & 0 & 1 \\
\hline asym.H & 1.0 & 0.4 & 0.7453 & $2 *$ & 0 & 0 & + & - & 0 & 0 & 1 \\
\hline Gauss & & 0.4 & 0.8128 & 3 & 0 & 0 & + & - & 0 & 0 & \\
\hline asym.L & 0.1 & 0.6 & 0.6609 & $2 *$ & 0 & 0 & + & $0_{*}$ & 0 & 0 & 2 \\
\hline asym.H & 1.0 & 0.6 & 0.6624 & $2 *$ & 0 & 0 & + & $0 *$ & 0 & 0 & 2 \\
\hline Gauss & & 0.6 & 0.7623 & 3 & 0 & 0 & + & - & 0 & 0 & \\
\hline asym.L & 0.1 & 0.8 & 0.5891 & $2 *$ & 0 & 0 & $\boldsymbol{T}_{*}$ & $0_{*}$ & $0_{*}$ & 0 & $4(1)$ \\
\hline asym.H & 1.0 & 0.8 & 0.5966 & $3 *$ & 0 & 0 & $\boldsymbol{+}_{*}$ & $0 *$ & $0_{*}$ & 0 & 4 \\
\hline Gauss & & 0.8 & 0.5795 & 0 & 0 & 0 & - & - & + & 0 & \\
\hline asym.L & 0.1 & 1.0 & 0.5350 & $2 *$ & 0 & 0 & $t_{*}$ & $0_{*}$ & $0_{*}$ & 0 & $4(1)$ \\
\hline asym.H & 1.0 & 1.0 & 0.5477 & $3 *$ & 0 & 0 & $\boldsymbol{+}_{*}$ & $0_{*}$ & $0_{*}$ & 0 & 4 \\
\hline Gauss & & 1.0 & 0.5796 & 1 & 0 & 0 & - & - & + & 0 & \\
\hline
\end{tabular}

(E) $\lambda=0.8$

\begin{tabular}{|l|ll|l}
\hline asym.L & 0.1 & 0.2 & 0.8922
\end{tabular}

$\mid \begin{array}{llll}2 * & 0 & 0\end{array}$

$+2$ 


\begin{tabular}{|c|c|c|c|c|c|c|c|c|c|c|c|}
\hline $\operatorname{asym} . \mathrm{H}$ & 1.0 & 0.2 & 0.8814 & $2 *$ & 0 & 0 & + & - & 0 & 0 & 1 \\
\hline Gauss & & 0.2 & 0.9148 & 3 & 0 & 0 & + & - & 0 & 0 & \\
\hline asym.L & 0.1 & 0.4 & 0.7701 & $2 *$ & 0 & 0 & 7 & 0 & 0 & 0 & 1 \\
\hline $\operatorname{asym} . \mathrm{H}$ & 1.0 & 0.4 & 0.7653 & $2 *$ & 0 & 0 & + & 0 & 0 & 0 & 1 \\
\hline Gauss & & 0.4 & 0.8394 & 3 & 0 & 0 & + & - & 0 & 0 & \\
\hline asym.L & 0.1 & 0.6 & 0.6809 & $2 *$ & 0 & 0 & + & 0 & 0 & 0 & 1 \\
\hline asym.H & 1.0 & 0.6 & 0.6824 & $2 *$ & 0 & 0 & + & 0 & 0 & 0 & 1 \\
\hline Gauss & & 0.6 & 0.7926 & 3 & 0 & 0 & + & 0 & 0 & 0 & \\
\hline asym.L & 0.1 & 0.8 & 0.6158 & $2 * \bullet$ & 0 & 0 & & $0 *$ & $0_{*}$ & 0 & $4(1)$ \\
\hline $\operatorname{asym} . \mathrm{H}$ & 1.0 & 0.8 & 0.6232 & $3 *$ & 0 & 0 & & $0_{*}$ & $0_{*}$ & 0 & 4 \\
\hline Gauss & & 0.8 & 0.6595 & 0 & 0 & 0 & - & - & + & 0 & \\
\hline asym.L & 0.1 & 1.0 & 0.5684 & $2 * \bullet$ & 0 & 0 & $t_{*}$ & $0_{*}$ & 0 & 0 & $3(1)$ \\
\hline asym.H & 1.0 & 1.0 & 0.5810 & $3 *$ & 0 & 0 & $t_{*}$ & $0_{*}$ & 0 & 0 & 3 \\
\hline Gauss & & 1.0 & 0.6796 & 1 & 0 & 0 & - & - & + & 0 & \\
\hline
\end{tabular}

\begin{tabular}{|c|c|c|c|c|c|c|c|c|c|c|c|}
\hline asym.L & 0.1 & 0.2 & 0.9000 & $2 *$ & 0 & 0 & 7 & $0_{*}$ & 0 & 0 & 2 \\
\hline asym.H & 1.0 & 0.2 & 0.8922 & $2 *$ & 0 & 0 & + & $0_{*}$ & 0 & 0 & 2 \\
\hline asym.L & 0.1 & 0.4 & 0.7834 & $2 *$ & 0 & 0 & + & 0 & 0 & 0 & 1 \\
\hline asym.H & 1.0 & 0.4 & 0.7786 & $2 *$ & 0 & 0 & + & 0 & 0 & 0 & 1 \\
\hline Gauss & & 0.4 & 0.8543 & 3 & 0 & 0 & + & 0 & 0 & 0 & \\
\hline Gauss & & 0.6 & 0.8126 & 3 & 0 & 0 & + & 0 & 0 & 0 & \\
\hline asym.L & 0.1 & 0.8 & 0.6424 & $2 * \bullet$ & 0 & 0 & $*$ & $0_{*}$ & $0_{*}$ & 0 & $4(1)$ \\
\hline asym.H & 1.0 & 0.8 & 0.6499 & $3 *$ & 0 & 0 & * & $0_{*}^{*}$ & $0_{*}$ & 0 & 4 \\
\hline Gauss & & 0.8 & 0.7395 & 0 & 0 & 0 & - & - & + & 0 & \\
\hline asym.L & 0.1 & 1.0 & 0.6017 & $2 * \bullet$ & 0 & 0 & 7 & $0_{*}$ & $0_{*}$ & 0 & $4(1)$ \\
\hline asym.H & 1.0 & 1.0 & 0.6144 & $3 *$ & 0 & 0 & + & $0 *$ & $0_{*}$ & 0 & 4 \\
\hline
\end{tabular}

Table 8: Comparison of the optimized results for the asymmetric and

Gaussian pulses for different parameters of efficiency $\left(k_{E}\right)$, regularization $(\lambda)$ and the strength of therapeutic action $(Q)$. Star symbol is used for the features - components of $\mathbf{s}_{\min }$ where the optimal solutions are different for symmetrical (line denoted as Gauss) or non-symmetrical (line denoted as "asym.") pulses. The rightmost column informs about the Hamming distance $d_{H, A-G}$ between the optimal configurations corresponding to the asymmetric or symmetric pulses, respectively (distance is equal to the total number of the star symbols within the line). In addition, the number in the parenthesis (included only for nonzero values) represents the Hamming distance between the asymmetric configurations.

\subsection{From discrete to continuous optimization on branched manifolds}

In this section we present two illustrative examples of the application of the hybrid optimization procedure which demonstrates significance of the consecutive corrections to the original discrete problem. The modification shows that the progress in the optimization is inseparable from the additional constraints and parametrizations.

The original settings given by Eq.(13) can be modified by means of newly introduced auxiliary six "weight" 
variables

$$
\begin{array}{lll}
\widetilde{h_{C T}}=h_{C T}\left(1+q_{h_{C T}} Q h_{C T} s_{h_{C T}} \psi_{C}\right), & \widetilde{g_{C C}}=g_{C C}\left(1+q_{g_{C C}} Q g_{C C} s_{g_{C C}} \psi_{C}\right), \\
\widetilde{g_{C B}}=g_{C B}\left(1+q_{g_{C B}} Q g_{C B} s_{g_{C B}} \psi_{C}\right), & \widetilde{h_{B T}}=h_{B T}\left(1+q_{h_{B T}} Q h_{B T} s_{h_{B T}} \psi_{B}\right), \\
\widetilde{g_{B C}}=g_{B C}\left(1+q_{g_{B C}} Q g_{B C} s_{g_{B C}} \psi_{B}\right), & \widetilde{g_{B B}}=g_{B B}\left(1+q_{g_{B} B} Q g_{B B} s_{g_{B}} \psi_{B}\right) .
\end{array}
$$

In order to preserve the original meaning of the unique scalar amplitude $Q$, the impact of optimization is redistributed on the non-uniform weights which satisfy constraint $\operatorname{Tr}(\mathbf{q})=q_{h_{C T}}+q_{g_{C C}}+q_{g_{C B}}+q_{h_{B T}}+q_{g_{B C}}+q_{g_{B B}}=$ $6, q_{\bullet}>q_{\text {ground }}>0$. The weighting is reflected by the modified penalty term $\lambda Q \mathbf{q} \cdot \mathbf{s}=\lambda Q\left(q_{h_{C T}} s_{h_{C T}}+q_{g_{C C}} s_{g_{C C}}\right.$ $\left.+q_{g_{C B}} s_{g_{C B}}+q_{h_{B T}} s_{h_{B T}}+q_{g_{B C}} s_{g_{B C}}+q_{g_{B B}} s_{g_{B B}}\right)$. The optimization constraints which delimit q components can be satisfied by considering specific parametrization. It can be represented by the one-dimensional manifold $M_{1} \equiv\left\{\mathbf{s} \in \Omega, \mathbf{q}(\eta) \in \mathbb{R}_{6}, \eta \in(0,1) \subset \mathbb{R}\right\}$ with the branches

$$
\begin{aligned}
& q_{h_{C T}}(\eta)=q_{\text {ground }}+\left(1-q_{\text {ground }}\right) 6 \eta^{5}, \\
& q_{g_{C C}}(\eta)=q_{\text {ground }}+\left(1-q_{\text {ground }}\right) 30 \eta^{4}(1-\eta), \\
& q_{g_{C B}}(\eta)=q_{\text {ground }}+\left(1-q_{\text {ground }}\right) 60 \eta^{3}(1-\eta)^{2}, \\
& q_{h_{B T}}(\eta)=q_{\text {ground }}+\left(1-q_{\text {ground }}\right) 60 \eta^{2}(1-\eta)^{3}, \\
& q_{g_{B C}}(\eta)=q_{\text {ground }}+\left(1-q_{\text {ground }}\right) 30 \eta(1-\eta)^{4} \\
& q_{g_{B B}}(\eta)=q_{\text {ground }}+\left(1-q_{\text {ground }}\right) 6(1-\eta)^{5}
\end{aligned}
$$

parametrized by the auxiliary real variable $\eta \in\langle 0,1\rangle$ and constant $q_{\mathrm{groun}} \in\langle 0,1\rangle$. We note, for later comparisons, that former discrete optimization corresponding to the uniform weighting $q_{\bullet}=1$ performed for the asymmetric pulses and $k_{E}=1, Q=0.4, \lambda=0.2$ leads to $\mathbf{s}_{\min }=[2,-1,0,1,-1,1,0]$ corresponding to $f_{\min }=0.666259$. Extending the search space on $M_{1}$ parametrized by $q_{\text {ground }}=0.2$, using the Ridders' variant of Dekker-Brent method [60] applied to dimension $\eta \in\langle 0,1\rangle$ leads to the modified $\mathbf{s}_{\text {min }}^{M_{1}}=[2,-1,1,1,-1,1,0]$ but lower $f_{\text {min }}^{M_{1}}=0.5266$ obtained for $\eta_{\text {min }}^{M_{1}} \simeq 0.583$. In such case, the highest optimal weight $q_{g_{C B}, \min }^{M_{1}} \simeq 1.85$ is posed on the OC-OB interaction term while the lowest weight $q_{g_{B B}, \min }^{M_{1}}=0.2605$ loses its meaning by being present simultaneously with the multiplier $s_{g_{B B}, \min }^{M_{1}}=0$.

It should be emphasized that the highest weight is consistent with the central role of $s_{g_{C B}}^{M_{1}}$ in coincidence with the findings of the synergistic concept (see sec 6.2). As there is no smooth transition to the uniform weighting for $M_{1}, q_{\text {ground }}<1$, we introduce the alternative manifold $M_{2}$ by $\mathbf{q}^{M_{2}}(\eta) \equiv q_{\text {ground }}[1,1,1,1,1,1]+(1-$ $\left.q_{\text {ground }}\right) 6\left[1, \eta, \eta^{2}, \eta^{3}, \eta^{4}, \eta^{5}\right] /\left(1+\eta+\eta^{2}+\eta^{3}+\eta^{4}+\eta^{5}\right)$ to reach $q_{\bullet}^{M_{2}}=1$ in the limit $\eta_{\text {min }}^{M_{2}}=\eta \rightarrow 1$. However, in this case the optimization routine has determined relatively shallow $f_{\text {min }}^{M_{2}}=0.6608$ corresponding to the string $\mathbf{s}_{\min }^{M_{2}}=[2,-1,0,1,-1,1,0]$ accompanied by $\eta_{\min }^{M_{2}}=0.86$ and weights $\mathbf{q}_{\text {min }}^{M_{2}} \simeq[1.30,1.15,1.03$, $0.92,0.83,0.75]$.

\subsection{Synergistic quantification of optimization results}

The optimization task that we studied clearly demonstrates that the parameters do not act independently to each other and the optimum depends on the simultaneous effect of the parametric combination. In the next, we 
put the multi-parametric optimization outputs into the context of existing synergistic concepts as conceived in the biomedical and farmacological studies of the simultaneous effects of therapeutic drugs (see e.g. [61, 62]). Despite many alternative measures and approaches have been already proposed, the synergistic quantification of the optimization outputs is not straightforward. The generalization we made is inspired by the form of classic Loewe's additivity principle [63].

Before performing quantification of the optimization of the parametric pairs, we introduce reduced string $\hat{\mathbf{s}}$ with the six enumerated components $\hat{s}_{1}=s_{h_{C T}}, \hat{s}_{2}=s_{g_{C C}}, \hat{s}_{3}=s_{g_{C B}}, \hat{s}_{4}=s_{h_{B T}}, \hat{s}_{5}=s_{g_{B C}}, \hat{s}_{6}=s_{g_{B B}}$. They allow to construct the sets of pairs $\hat{\Omega}_{i j}=\left\{\left\{\hat{s}_{i}, \hat{s}_{j}\right\} ; \hat{s}_{k}=0 \forall k \neq i, \forall k \neq j\right\}$. The extraordinary $s_{I}$ (its zero value does not mean the absence of the pulse influence) is excluded from the enumeration, nevertheless, its optimization effect is considered as well; see Eq. 35 below. Selection of $\hat{\Omega}_{i j}$ is consistent with the fact that pairwise synergy makes sense only for nonzero pairs $\hat{s}_{i}, \hat{s}_{j}$. The corresponding scalar measures may be arranged into $6 \times 6$ matrix of the generalized combination indices

$$
C I f_{i j}=\frac{\hat{f}_{\text {min }, i j}}{2 \hat{f}_{\text {min }, i i}}+\frac{\hat{f}_{\text {min }, i j}}{2 \hat{f}_{\text {min }, j j}}=\hat{f}_{\text {min }, i j} \underbrace{\left(\frac{2 \hat{f}_{\text {min }, i i} \hat{f}_{\text {min }, j j}}{\hat{f}_{\text {min }, i i}+\hat{f}_{\text {min }, j j}}\right)^{-1}}_{\text {harmonic mean }},
$$

where

$$
\hat{f}_{\text {min }, i j}=\min _{\left\{\hat{s}_{i}, \hat{s}_{j}\right\} \in \hat{\Omega}_{i j}, s_{I} \in \Omega^{(4)}} f(\ldots) .
$$

Here, the single parameter effects are involved in the diagonal elements $\hat{f}_{m i n, i i}, \hat{f}_{m i n, j j}$, while the cumulative effects of the pairs are included in $\hat{f}_{m i n, i j}$. There is an obvious freedom of the choice of the prefactor [see Eq.[34]], however, as one may check, only the multiplication of both summands by $1 / 2$ leads to the harmonic mean normalization of $\hat{f}_{\text {min }, i j}$.

The properties $C I f_{i j}=C I f_{j i}, \forall i, j$ (symmetry); $C I f_{i i}=1, \forall i$ (unit diagonal as a referential value corresponding to monoparametric effects); $C I f_{i j}>0$ [implicated by $f(\ldots)>0$ ] $C I f_{i j} \leq 1 \forall i, j$ can be checked easily. At this point, it is also important to note the basic difference between the effects of monotherapy and the effects caused by the optimization of individual parameters. In agreement with the original conception of the combination index, lower index value means higher synergy of the parametric pair.

To avoid detailed comparison of the pairs, we turn to the information comprised in $I C f_{i}^{\prime}=(1 / 5) \sum_{j=1 ; j \neq i}^{6} I C f_{i j}$. In the correspondence with the standard Loewe's formulation, the higher the synergy, the lower $C I f_{i j}$ (or $C I f_{i}^{\prime}$ ) is obtained. The results of the numerical calculations of the six components of $I C f_{i}^{\prime}(Q)$ are depicted in Fig 9 . They uncover the highest cumulative synergistic effect of $\hat{s}_{3}=s_{g_{C B}}$ with another parameters. The identification of the central role of $s_{g_{C B}}$ in the optimization process is in the qualitative agreement with Table 8 , where the nonzero $s_{g_{C B}}$ persists up to the high $\lambda>0$ considered. 


\section{Conceptual model, indirect environmental manipulation}

The key aspect of our approach is the formal separation of the system into the environmental and cancerous compartments (populations), as well as the identification of the parameters appropriate for indirect environmental therapeutic manipulation. Specific information contained in the results delivered exclusively by simulations is hardly transferable between alternative models. The transmission can be facilitated by the generalization obtained by filtering, comparative analysis and synthesis of some particular information contained in the population models.

The way how to do it is the conceptualization based on general and abstract dynamic system for the pair of the population vectors $(\mathcal{T}(t), \mathcal{E}(t))$, where $\mathcal{T}(t)$ describes the tumoral and $\mathcal{E}(t)$ environmental dynamics, respectively. The parametric selection is involved in the constant tuple $\chi_{\mathcal{E}}$ (see Eq. (9) as particular example). Then, the pair of the corresponding rate functions $\left[\mathcal{R}_{T}\left(\mathcal{T}, \mathcal{E}\left[\chi_{\mathcal{E}}\right]\right), \mathcal{R}_{E}\left(\mathcal{T}, \mathcal{E}\left[\chi_{\mathcal{E}}\right]\right)\right]$ characterizes the original autonomous ODEs system

$$
\frac{d}{d t}\left(\begin{array}{c}
\mathcal{T} \\
\mathcal{E}
\end{array}\right)=\left(\begin{array}{c}
\mathcal{R}_{\mathcal{T}}\left(\mathcal{T}, \mathcal{E}\left[\chi_{\mathcal{E}}\right]\right) \\
\mathcal{R}_{\mathcal{E}}\left(\mathcal{T}, \mathcal{E}\left[\chi_{\mathcal{E}}\right]\right)
\end{array}\right)
$$

The exogenous variant of ODEs system belongs to the respective substitutions $\chi_{\mathcal{E}} \rightarrow \widetilde{\chi}_{\mathcal{E}}(t, \mathbf{s}), \mathcal{E}\left[\chi_{\mathcal{E}}\right] \rightarrow$ $\mathcal{E}\left[\widetilde{\chi}_{\mathcal{E}}(t, \mathbf{s})\right], \mathcal{R}_{X}\left(\mathcal{T}, \mathcal{E}\left[\chi_{\mathcal{E}}\right]\right) \rightarrow \widetilde{\mathcal{R}}_{X}\left(\mathcal{T}, \mathcal{E}\left[\widetilde{\chi}_{\mathcal{E}}(t, \mathbf{s})\right]\right)$ for $\mathbf{s} \in \Omega$ (see Eq. 10 ) and $X \in\{\mathcal{T}, \mathcal{E}\}$. Formally, the solutions $\left[\mathcal{T}\left(t, \mathbf{s}, \mathcal{T}_{\text {init }}, \mathcal{E}_{\text {init }}\right), \mathcal{E}\left(t, \mathbf{s}, \mathcal{T}_{\text {init }}, \mathcal{E}_{\text {init }}\right)\right]$ for given initial condition $\left[\mathcal{T}\left(t_{\text {init }}\right), \mathcal{E}\left(t_{\text {init }}\right)\right] \equiv\left[\mathcal{T}_{\text {init }}, \mathcal{E}_{\text {init }}\right]$ with $t_{\text {init }} \leq t_{S}$ can be obtained. Subsequently, the adverse effects of the tumoral dynamics along the path segment

$$
\mathcal{P}_{\mathcal{T}, \mathcal{E}}\left(t_{S}, t_{E}, \mathbf{s}\right) \equiv\left\{\left[\mathcal{T}\left(t, \mathbf{s}, \mathcal{T}_{\text {init }}, \mathcal{E}_{\text {init }}\right), \mathcal{E}\left(t, \mathbf{s}, \mathcal{T}_{\text {init }}, \mathcal{E}_{\text {init }}\right)\right], \forall t \in\left\langle t_{S}, t_{E}\right\rangle\right\}
$$

are quantifiable by the objective function

$$
f\left(t_{S}, t_{E}, \mathbf{s}\right)=\mathcal{F}\left\{\mathcal{P}_{\mathcal{T}, \mathcal{E}}\left(t_{S}, t_{E}, \mathbf{s}\right)\right\}
$$

where $\mathcal{F}\{$.$\} stands for some real-valued functional. In particular cases, that we have already discussed (see$ Eq. (18)), the special emphasis has been placed on the boundary interval values. Again, as in the case of specific formulation in sec 2 , the optimal therapy $\mathbf{s}_{\min }$ is determined by Eq. 20 .

\section{Conclusion}

In this article we present a global strategy that combines sensitivity analysis and global optimization approach which contrasts with the single parameter (local) investigations. As an intriguing aspect of our holistic view we see the synthesis of several different research directions. Substantial nonlinearity of ODE system is studied using multi-parametric pulses of symmetric and asymmetric form. In general terms, our approach provides a mapping between continuous problem formulated as ODEs and discrete problem where the ordering of the solutions according to the values of the respective objective function matters. Apart from the optimal therapeutic procedure itself, we are interested in the analysis of the sensitivity of differential equation systems to the complex parametric changes to which the potential therapies can be targeted. The results support potential benefits of our approach not only for monotherapies but also for the combined treatments. By incorporating synergistic 
measures of the parametric pairs, progress has been made in the model-level understanding of the complex systemic responses to the multi-dimensional pulses. It seems that many models of biological systems could be studied in a similar way regardless of the area of application. From a biological/pharmacological point of view, we do not follow conventional line of direct elimination of cancer cells; instead, more emphasis is given to the indirect effect achieved by the optimized manipulation with the tumoral environment. In addition, we point out that the results of our discrete combinatorial approach reveal many possible alternatives of restoring the balance between myeloma and the environment, as opposed to achieving higher optimality for a narrow set of specialized therapies.

Biological relevance of here analyzed MM system derives from the models by Komarova et al. [8] and Koenders and Saso [9]. This model for studying tumor - bone interactions is built in accordance with the general parsimony framework [64] which captures the fundamentals of the system. Presented approaches contribute to the understanding of the respective nonlinear ODEs by analyzing their complex responses. The works which focus on the problem of cancer treatment and its modeling can be roughly divided along two principal therapeutic targets: the tumor or its environment, respectively [28]. Our present work is in line with environmental aspect of cancer progression which is profoundly stressed in evolutionary and ecological models of cancer [65, 66, 67, 12]. We hope that the methodology presented in this work will be extended to take into account intratumor heterogeneity [68, 69, 70], e.g. cell-to-cell heterogeneity at the MM level [71, 30, 36]. The indirect, environmentally oriented therapies can cause the erosion of the habitats of the cancer clones, or, eventually, to decrease the probability of drug resistance. Many promising and innovated therapies exploit indirect therapeutic effects, such as the cell cycle manipulation, influencing or remodeling of vascular networks [72, 73]. More specifically, in Ref. [74] the authors identified indirect antimyeloma mechanisms preventing bone resorption [74]. Another example which should be mentioned in this context is immunotherapy [50, 51, 75, 76], where particular emphasis is placed on tumor-associated macrophages and cancer-associated fibroblasts as the determinants of the tumor microenvironment. The recent literature [77] reminds that homeostasis of normal bone tissue formed by OBs, OCs and osteocytes is regulated predominantly by osteocyte cells derived from OBs. In the case of MM, homeostasis is impaired, leading to the bone resorption, pathogenic lesions and tumor expansion. We assume that this type of knowledge can be appropriately integrated into the three-component form of the environmental population with eventual therapeutic influence on tumorigenesis.

\section{Acknowledgments}

This work was supported by (a) APVV-15-0485 by Slovak Research and Development Agency;(b) VEGA No. 1/0250/18; (c) VEGA 1/0156/18 by Scientific Grant Agency of the Ministry of Education of Slovak Republic. 


\section{A Appendices}

\section{A.1 Robustness of the optimum, reoptimization in the periodic environment}

The therapies can be delivered in periodic or other appropriate manner [48, 59] instead of in one time only. As an alternative to focusing on the optimal number of the pulse repeats, we investigate the feasibility and robustness of the periodic extension of the results achieved for single pulse [see sec 3.3] without reoptimization for the periodic conditions. The extension represents a kind of myopic, short-term strategy [78].

Technically, the extension we applied is based on $\mathbf{s}_{\min }$ and period $t_{E}-t_{S}$. The transient, not purely periodic, $C(t), B(t), T(t), J(t)$ responses are obtained when the integration of Eqs.(1), (2) is performed for the sequence of the joint intervals $\left\langle t_{0}^{(\text {per })}(m), t_{1}^{(\text {per })}(m)\right\rangle$, where $m=0,1,2, \ldots$ are indices of the periodic shifts $t_{0}^{(\text {per })}(m)=$ $t_{0}+m\left(t_{E}-t_{S}\right), t_{1}^{(p e r)}(m)=t_{1}+m\left(t_{E}-t_{S}\right)$ in the locations of the peaks replacing $t_{0}, t_{1}$ in the functions $\psi\left(t, t_{0} \rightarrow t_{0}^{(\text {per })}(m), \ldots\right)$ and $\psi\left(t, t_{1} \rightarrow t_{1}^{(p e r)}(m), \ldots\right)$.

From Figs.(10) and (11) we see that despite the optimization has not been performed in the periodic case and only suboptimal $\mathbf{s}_{\min }$ has been used, it turns out that the therapeutic interventions obtained are sufficient to keep tumors within safe limits.

\section{A.2 Stability of integrators}

In the numerical ODE analysis, the explicit integration methods assume that almost any numerical precision can be achieved by selecting small enough integration step. Therefore, if the explicit methods, such as the above mentioned RK4 are applied, great care must be taken to guarantee the convergence and the asymptotic properties of iterates, as these may exhibit strong dependence on the integration step size. More specifically, the problem known as ODE stiffness occurs in the cases where approximate errors of the numerical method cannot be sufficiently suppressed regardless of how small the integration step is. Therefore, robust implicit integrators need to be used to verify solutions obtained using explicit methods. For the ODE application described above we perform combined problem-specific testing using the overall integration-optimization procedure instead of purely numerical integration without locating optima. The aim of the combined testing [see Fig,12] is to examine the persistence of $\min f$ (and the related metrics $D f_{\min }$ ), as well as revealing potential stiffness artifacts.

In Table 9 we list the thresholds in the parameter $\Delta t$ which indicate the onset of instabilities of the respective integrators. The numerical findings for RK4 are provided along with Euler predictor-corrector (PEC) and PECECE variants with recurrent corrector steps. Both alternative integrators can be classified as implicit due to the incorporation of the trapezoidal rule. By comparing the tabulated thresholds obtained for several $Q$ we have obtained a universal lower estimate of $\Delta t \sim \mathcal{O}(0.01)$. It is obvious that the use of this or smaller step size does not pose the risk of substantial stiffness errors for any of the above methods, which agree in $\mathbf{s}_{\min }$. This means that our original choice of $\Delta t=5 \times 10^{-4}$ for RK4 provided reliable results. 


\begin{tabular}{|l||ll|ll|ll|}
\hline$Q$ & $\begin{array}{l}\text { RK4 } \\
\text { rtol } \\
=10^{-3}\end{array}$ & $\begin{array}{l}\text { RK4 } \\
\text { rtol } \\
=2 \times 10^{-3}\end{array}$ & $\begin{array}{l}\text { PEC } \\
\text { rtol } \\
=10^{-3}\end{array}$ & $\begin{array}{l}\text { PEC } \\
\text { rtol } \\
=2 \times 10^{-3}\end{array}$ & $\begin{array}{l}\text { PECECE } \\
\text { rtol } \\
=10^{-3}\end{array}$ & $\begin{array}{l}\text { PECECE } \\
\text { rtol } \\
=2 \times 10^{-3}\end{array}$ \\
\hline 0.1 & 0.496 & 0.966 & 0.530 & 1.036 & 0.506 & 1.025 \\
0.4 & 0.116 & 0.219 & 0.146 & 0.279 & 0.146 & 0.291 \\
1.0 & 0.024 & 0.045 & 0.042 & 0.077 & 0.042 & 0.077 \\
3.0 & 0.022 & 0.038 & 0.158 & 0.248 & 0.276 & 0.303 \\
\hline
\end{tabular}

Table 9: The table contains list of the threshold values of the parameter $\Delta t$ defined as the lowest values for which the relative change of $\min f$ reaches at least the predefined levels of relative tolerance $r$ tol $=10^{-3}$ (or $2 \times 10^{-3}$ ) with respect to the reference (saturated) value of min $f$ obtained for sufficiently small $\Delta t=5 \times 10^{-4}$. We see that PEC stability exceeds those of RK4, moreover, adding another iteration loop within PECECE seems to be even redundant for given parameters and intervals studied. The advantage of implicit methods is more obvious at $Q=1$, where the instabilities are stronger (see Fig.5). The calculations are performed for $n_{0}=0$.

\section{References}

[1] L. Preziosi (Ed.), Cancer Modelling and Simulation, Chapman\&Hall/CRC London, 2003.

[2] R. A. Gatenby, P. K. Maini, Mathematical oncology: Cancer summed up, Nature 421 (2003) 321.

[3] T. Roose, S. J. Chapman, P. K. Maini, Mathematical models of avascular tumor growth, SIAM Rev. 49 (2007) 179-208.

[4] N. Bellomo, N. K. Li, P. K. Maini, On the foundations of cancer modelling: Selected topics, speculations, and perspectives, Math. Mod. Meth. Appl. S. 18 (2008) 593-646.

[5] M. Transtrum, P. Qiu, Model reduction by manifold boundaries, Phys. Rev. Lett. 113 (2014) 098701.

[6] S. Sanga, J. Sinek, H. Frieboes, M. Ferrari, J. Fruehauf, V. Cristini, Mathematical modeling of cancer progression and response to chemotherapy, Expert Rev Anticancer Ther. 6 (10) (2006) 1361-1376.

[7] D. Raman, J. Anderson, A. Papachristodoulou, Delineating parameter unidentifiabilities in complex models, Phys. Rev. E 95 (2017) 032314.

[8] S. Komarova, R. Smith, S. Dixon, S. Sims, L. Wahl, Mathematical model predicts a critical role for osteoclast autocrine regulation in the control of bone remodeling, Bone 33 (2003) 206-215.

[9] M. Koenders, R. Saso, A mathematical model of cell equilibrium and joint cell formation in multiple myeloma, J. Theor. Biol. 390 (2016) 73 - 79. 
[10] R. Yafia, Dynamics analysis and limit cycle in a delayed model for tumor growth with quiescence, Nonlinear Analysis: Modeling and Control 11 (2006) 95-110.

[11] R. A. Gatenby, T. L. Vincent, An evolutionary model of carcinogenesis, Cancer Res. 63 (19) (2003) 62126220.

[12] R. A. Gatenby, A change of strategy in the war on cancer, Nature 459 (2009) 508-509.

[13] R. A. Gatenby, A. S. Silva, R. J. Gillies, B. R. Frieden, Adaptive therapy, Cancer Res. 69 (2009) 4894-4903.

[14] R. J. Gillies, D. Verduzo, R. A. Gatenby, Evolutionary dynamics of carcinogenesis and why targeted therapy does not work, Nat. Rev. Cancer 12 (2012) 487-493.

[15] H. W. Engl, C. Flamm, P. Kügler, J. Lu, S. Müller, P. Schuster, Inverse problems in systems biology, Inverse Problems 25 (12) (2009) 123014.

[16] R. Gutenkunst, J. Waterfall, F. Casey, K. Brown, C. Myers, J. Sethna, Universally sloppy parameter sensitivities in systems biology models, PLOS Computational Biology 3 (10) (2007) 1-8.

[17] B. Ingalls, Sensitivity analysis: from model parameters to system behaviour, Essays In Biochemistry 45 (2008) 177-194.

[18] S. Marino, I. B. Hogue, C. J. Ray, D. E. Kirschner, A methodology for performing global uncertainty and sensitivity analysis in systems biology, J. Theor. Biol. 254 (1) (2008) $178-196$.

[19] S. Sahle, P. Mendes, S. Hoops, U. Kummer, A new strategy for assessing sensitivities in biochemical models, Philos Trans A Math Phys Eng Sci 366 (1880) (2008) 3619-3631.

[20] Z. Zi, Sensitivity analysis approaches applied to systems biology models, IET Systems Biology 5 (6) (2011) 336-6.

[21] A. Babtie, P.Kirk, M. Stumpf, Topological sensitivity analysis for systems biology, Proc Natl Acad Sci U S A 111 (2014) 18507-18512.

[22] X.-Y. Zhang, M. Trame, L. Lesko, S. Schmidt, Sobol sensitivity analysis: A tool to guide the development and evaluation of systems pharmacology models, CPT Pharmacometrics Syst. Pharmacol. 4 (2015) 69-79.

[23] F. Ferretti, A. Saltelli, S. Tarantola, Trends in sensitivity analysis practice in the last decade, Science of The Total Environment 568 (2016) 666 - 670.

[24] E. Borgonovo, E. Plischke, Sensitivity analysis: A review of recent advances, European Journal of Operational Research 248 (3) (2016) 869 - 887.

[25] D. Hadjidakis, I. Androulakis, Bone remodeling, Annals of the New York Academy of Sciences 1092 (1) (2006) 385-396. 
[26] D. Dingli, F. A. C. C. Chalub, F. C. Santos, S. V. Segbroeck, J. M. Pacheco, Cancer phenotype as the outcome of an evolutionary game between normal and malignant cells, Br. J. Cancer 101 (2009) 11301136.

[27] M. Ryser, N. Nigam, S. Komarova, Mathematical modeling of spatio-temporal dynamics of a single bone multicellular unit, Journal of Bone and Mineral Research 24 (5) (2009) 860-870.

[28] B. P. Ayati, C. M. Edwards, G. F. Webb, J. P. Wikswo, A mathematical model of bone remodeling dynamics for normal bone cell populations and myeloma bone disease, Biol. Direct 5 (2010) 1-28.

[29] M. Qiao, D. Wu, M. Carey, X. Zhou, L. Zhang, Multi-scale agent-based multiple myeloma cancer modeling and the related study of the balance between osteoclasts and osteoblasts, PLoS one 12 (10) (2015) e0143206.

[30] A. Bouchnita, F. Belmaati, R. Aboulaich, M. Koury, V. Volpert, A hybrid computation model to describe the progression of multiple myeloma and its intra-clonal heterogeneity, Computation 5 (1) (2017) pp.16.

[31] M. Peterson, M. Riggs, A physiologically based mathematical model of integrated calcium homeostasis and bone remodeling, Bone 46 (1) (2010) 49-63.

[32] A. Zomas, E. Terpos, E. Katodritou, A. Symeonidis, S. Delimpasi, A. Pouli, T. P. Vassilakopoulos, E. Michalis, S. Giannouli, Z. Kartasis, A. Christoforidou, K. Kokoviadou, E. Hatzimichael, D. Gika, C. Megalakaki, M. Papaioannou, M.-C. Kyrtsonis, K. Konstantopoulos, M. A. Dimopoulos, E. Kastritis, Hypercalcemia remains an adverse prognostic factor for newly diagnosed patients with symptomatic multiple myeloma in the era of novel anti-myeloma therapies, independently of age, iss stage and treatment type: An analysis of 2129 patients, Blood 124 (2014) 2113.

[33] K. Kupisiewicz, Biological aspects of altered bone remodeling in multiple myeloma and possibilities of pharmacological intervention, Dan Med Bull. 58 (5) (2011) B4277.

[34] T. L. Andersen, P. Boissy, T. E. Sondergaard, K. Kupisiewicz, T. Plesner, T. Rasmussen, J. Haaber, S. Kolvraa, J. M. Delaisse, Osteoclast nuclei of myeloma patients show chromosome translocations specific for the myeloma cell clone: a new type of cancer-host partnership?, J. Pathol. 211 (2007) 10-17.

[35] V. Lemaire, F. L. Tobin, L. D. Greller, C. R. Cho, L. J. Suva, Modeling the interactions between osteoblast and osteoclast activities in bone remodeling, J. Theor. Biol. 229 (3) (2004) 293 - 309.

[36] M. Tang, R. Zhao, H. van de Velde, J. G. Tross, C. Mitsiades, S. Viselli, R. Neuwirth, D. Esseltine, K. Anderson, I. M. Ghobrial, J. F. S. Miguel, P. G. Richardson, M. H. Tomasson, F. Michor, Myeloma cell dynamics in response to treatment supports a model of hierarchical differentiation and clonal evolution, Clinical Cancer Research 22 (16).

[37] K. F. Noubissi, B. M. Ogle, Cancer cell fusion: Mechanisms slowly unravel, Int. J. Mol. Sci. 17 (2016) 1587. 
[38] S. C. Searles, E. K. Santosa, J. D. Bui, Cell-cell fusion as a mechanism of dna exchange in cancer, Oncotarget 9 (2018) 6156-6173.

[39] M. Costa, J. Boldrini, Conflicting objectives in chemotherapy with drug resistance, Bulletin of Mathematical Biology 59 (4) (1997) 707-724.

[40] K. Miettinen, M. Mäkelä, On scalarizing functions in multiobjective optimization, OR Spectrum 24 (2) (2002) 193-213.

[41] M. Ehrgott, A discussion of scalarization techniques for multiple objective integer programming, Annals of Operations Research 147 (1) (2006) 343-360.

[42] G. Beliakov, H. Sola, T. Sanchez, A Practical Guide to Averaging Functions, 1st Edition, Springer Publishing Company, Incorporated, 2015.

[43] M. Claesen, B. D. Moor, Hyperparameter search in machine learning, CoRR abs/1502.02127. URL http: //arxiv.org/abs/1502.02127

[44] M. Wistuba, N. Schilling, L. Schmidt-Thieme, Hyperparameter search space pruning - a new component for sequential model-based hyperparameter optimization, in: A. Appice, P. P. Rodrigues, V. Santos Costa, J. Gama, A. Jorge, C. Soares (Eds.), Machine Learning and Knowledge Discovery in Databases: European Conference, ECML PKDD 2015, Porto, Portugal, September 7-11, 2015, Proceedings, Part II, Springer International Publishing, 2015, pp. 104-119.

[45] U. Heider, L. Hofbauer, I. Zavrski, M. Kaiser, C. Jakob, O. Sezer, Novel aspects of osteoclast activation and osteoblast inhibition in myeloma bone disease, Biochemical and Biophysical Research Communications 338 (2) (2005) $687-693$.

[46] J. Butcher, Numerical Methods for Ordinary Differential Equations, Second Edition, John Wiley \& Sons, Chichester, 2016.

[47] H. L. Thi, T. P. Dinh, H. Le, X. Vo, Dc approximation approaches for sparse optimization, European Journal of Operational Research 244 (1) (2015) $26-46$.

[48] J. Foo, F. Michor, Evolution of resistance to targeted anti-cancertherapies during continuous and pulsed administration strategies, PLoS Computational Biology 5 (11) (2009) e1000557.

[49] D. Nichol, P. Jeavons, A. G. Fletcher, R. A. Bonomo, P. K. Maini, J. L. Paul, R. A. Gatenby, A. Anderson, J. G. Scott, Steering evolution with sequential therapy to prevent the emergence of bacterial antibiotic resistance, PLoS Comput Biol 11 (9) (2015) e1004493.

[50] A. Cappuccio, F. Castiglione, B. Piccoli, Determination of the optimal therapeutic protocols in cancer immunotherapy, Math Biosci. 209 (1) (2007) 1-13. 
[51] F. Castiglione, B. Piccoli, Optimal control in a model of dendritic cell transfection cancer immunotherapy, Bulletin of Mathematical Biology 68 (2) (2006) 255âĂŞ-274.

[52] A. Wald, Statistical Decision Functions, John Wiley, New York, 1950.

[53] G. Parmigiani, L. Inoue, Decision Theory: Principles and Approaches, John Wiley and Sons, United Kingdom, 2009.

[54] H. d'Albis, E. Thibault, Optimal annuitization, uncertain survival probabilities, and maxmin preferences, Economics Letters 115 (2) (2012) 296 - 299.

[55] K. V. Deun, T. Wilderjans, R. V. den Berg, A. Antoniadis, I. V. Mechelen, A flexible framework for sparse simultaneous component based data integration, BMC Bioinformatics (2011) 448.

[56] S. Yaccoby, M. Wezeman, A. Henderson, M. Cottler-Fox, Q. Yi, B. Barlogie, J. Epstein, Cancer and the microenvironment: myelomaâĂŞosteoclast interactions as a model, Cancer Res. 64 (6) (2004) 2016-23.

[57] C. Edwards, J. Zhuang, G. Mundy, The pathogenesis of the bone disease of multiple myeloma, Bone 42 (6) (2008) 1007-1013.

[58] J. Brodie, I.Daubechies, C. Mol, D. Giannone, I. Loris, Sparse and stable markowitz portfolios, Proc Natl Acad Sci USA 106 (30) (2009) 12267-12272.

[59] R. Ananthakrishnan, P. Gona, Pharmacological modeling and biostatistical analysis of a new drug, Open Access Journal of Clinical Trials 2 (2010) 59-82.

[60] C. Ridders, Three-point iterations derived from exponential curve fitting, IEEE Transactions on Circuits and Systems 26 (8) (1979) 669-670.

[61] K. R. Roell, D. M. Reif, A. A. Motsinger-Reif, An introduction to terminology and methodology of chemical synergyâĂŤperspectives from across disciplines, Frontiers in Pharmacology 8 (2017) 158.

[62] A. Palmer, P. Sorger, Combination cancer therapy can confer benefit via patient-to-patient variability without drug additivity or synergy, Cell 171 (7) (2017) 1678-1691.

[63] S. Loewe, H. Muischnek, Effect of combinations: mathematical basis of the problem, Arch. Exp. Pathol. Pharmakol. 114 (1926) 313-326.

[64] P. Pivonka, S. Komarova, Mathematical modeling in bone biology: From intracellular signaling to tissue mechanics, Bone 47 (2010) 181-189.

[65] L. M. F. Merlo, J. W. Pepper, B. J. Reid, C. C. Maley, Cancer as an evolutionary and ecological process, Nat. Rev. Cancer 6 (2006) 924-935.

[66] A. R. A. Anderson, A. M. Weaver, P. T. Cummings, V. Quaranta, Tumor morphology and phenotypic evolution driven by selective pressure from microenvironment, Cell 127 (2006) 905-915. 
[67] M. Greaves, Darwinian medicine: a case for cancer, Nat. Rev. Cancer 7 (2007) 213-221.

[68] D. Wodarz, N. Komarova, Computational Biology of Cancer: Lecture notes and mathematical modeling, World Scientific, Singapore, 2005.

[69] D. Amor, R. Solé, Catastrophic shifts and lethal thresholds in a propagating front model of unstable tumor progression, Phys. Rev. E 90 (2014) 022710.

[70] D. Horvath, B. Brutovsky, Etiology of phenotype switching strategies in time varying environment, Phys. Lett. A 380 (13) (2016) 1267-1278.

[71] T. Paíno, B. Paiva, J. Sayagués, I. Mota, T. Carvalheiro, L. Corchete, I. Aires-Mejía, J. Pérez, M. Sanchez, P. Barcena, E. Ocio, L. San-Segundo, M. Sarasquete, R. García-Sanz, M. Vidriales, A. Oriol, M. Hernández, M. Echeveste, A. Paiva, J. Blade, J. Lahuerta, A. Orfao, M. Mateos, N. Gutiérrez, J. San-Miguel, Phenotypic identification of subclones in multiple myeloma with different chemoresistant, cytogenetic and clonogenic potential, Leukemia 29 (5) (2015) 1186-1194.

[72] A. Swierniak, M. Kimmel, J. Smieja, Mathematical modeling as a tool for planning anticancer therapy, European journal of pharmacology 625 (1-3) (2009) 108.

[73] H. Byrne, Dissecting cancer through mathematics: from the cell to the animal model, Nat Rev Cancer 10 (3) (2010) 221-230.

[74] N. Modi, S. Lentzsch, Bisphosphonates as antimyeloma drugs, Leukemia 26 (4) (2012) 589-594.

[75] L. de Pillis, W. Gu, A. Radunskaya, Mixed immunotherapy and chemotherapy of tumors: modeling, applications and biological interpretations, J. Theor. Biol. 238 (4) (2006) 841-862.

[76] R. Reisfeld, The tumor microenvironment: a target for combination therapy of breast cancer, Crit Rev Oncol. 18 (1-2) (2013) 115-133.

[77] E. Terpos, D. Christoulas, M. Gavriatopoulou, Biology and treatment of myeloma related bone disease, Metabolism 80 (2018) 80-90.

[78] J. Handa, Monetary Economics, Routledge, Taylor \& Francis group, London, New York, 2008. 

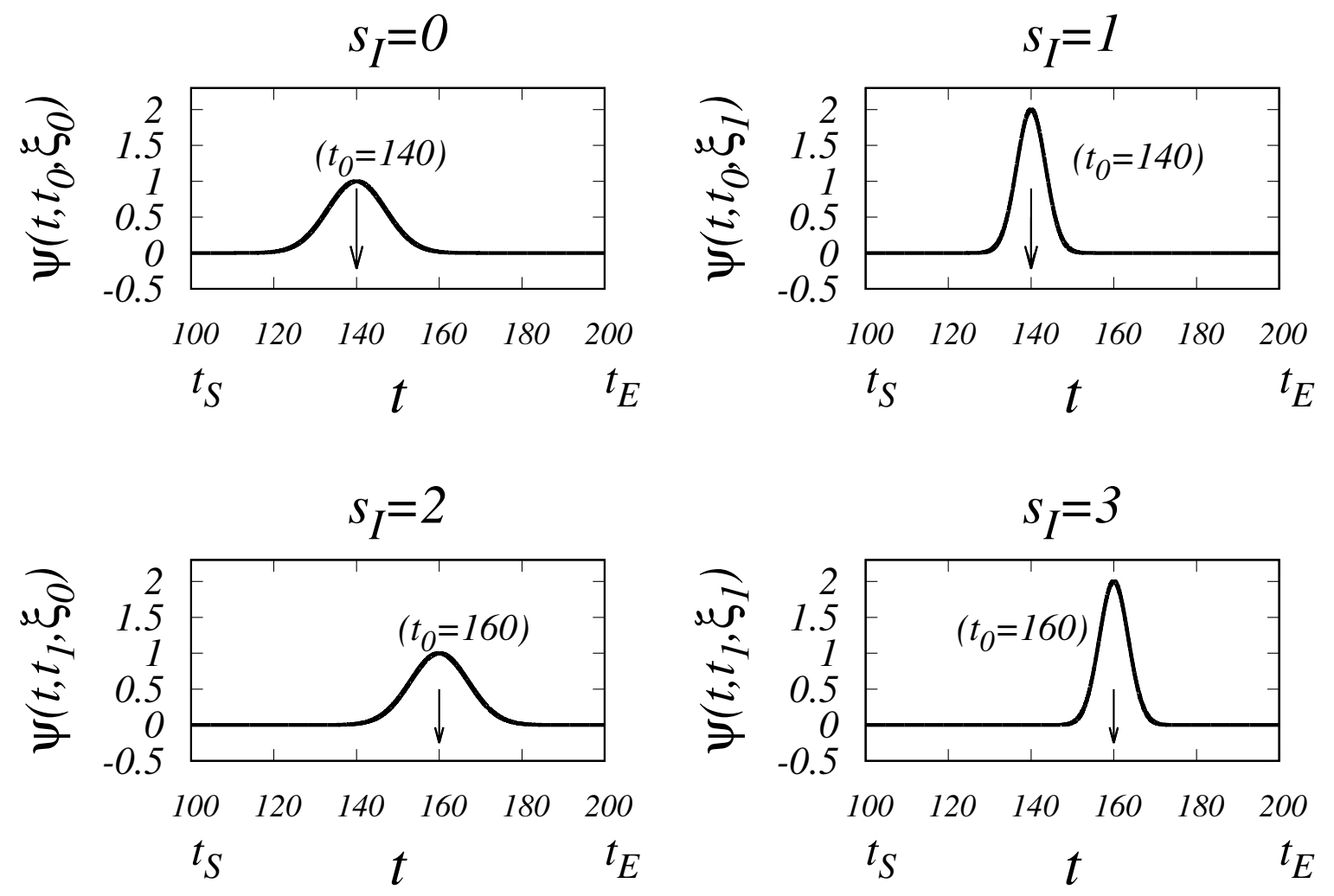

Figure 2: The dynamic elements of the sensitivity analysis generated by the translation and scaling of the arguments of the Gaussian pulses from Eq. (17). The calculation is based on the parameters $t_{0}, \xi_{0}, t_{1}, \xi_{1}, \sigma_{0}$ defined by Eqs. (25) and (26). The positions of $t=t_{0}$ are indicated by the vertical arrows. The examples of four child wavelets Eq. 16 used to construct $\Psi_{B}(t)$ (corresponding to $s_{I}=0$ ) and $\Psi_{C}\left(t, s_{I}\right)$ (for $s_{I} \in \Omega^{(4)}$ ). 

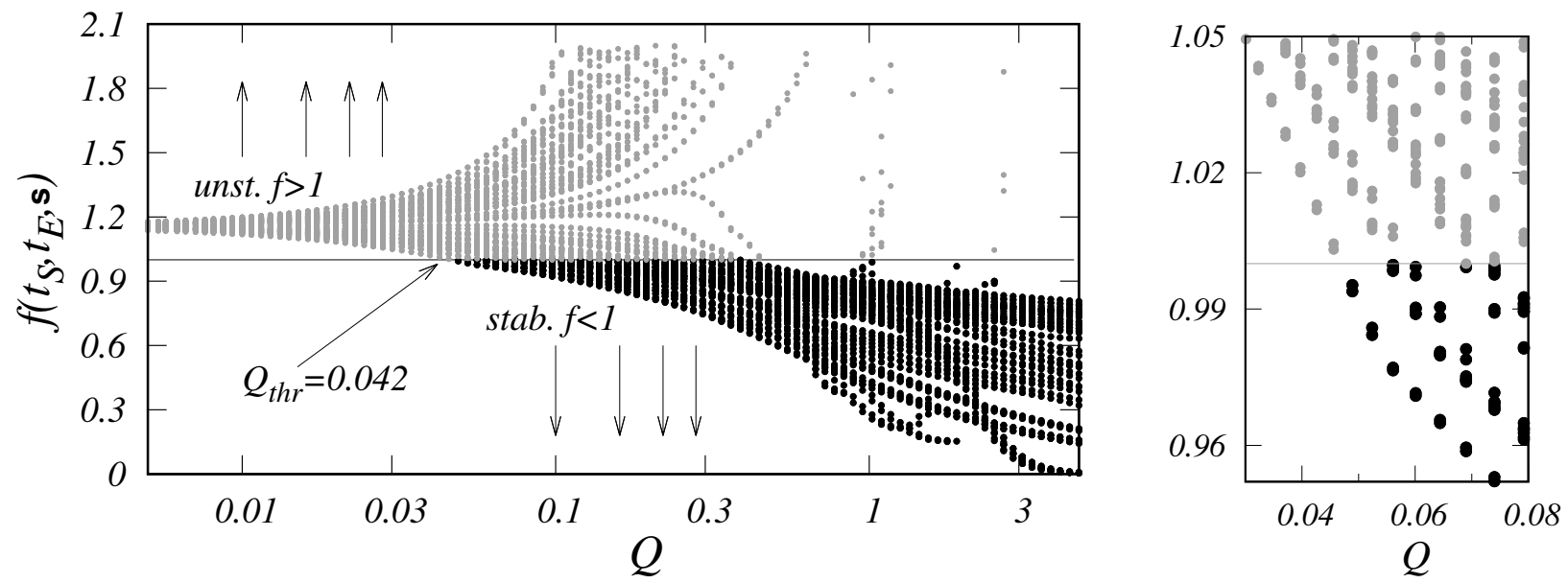

(a) $n_{0}=0$

(b) $n_{0}=0$ detail
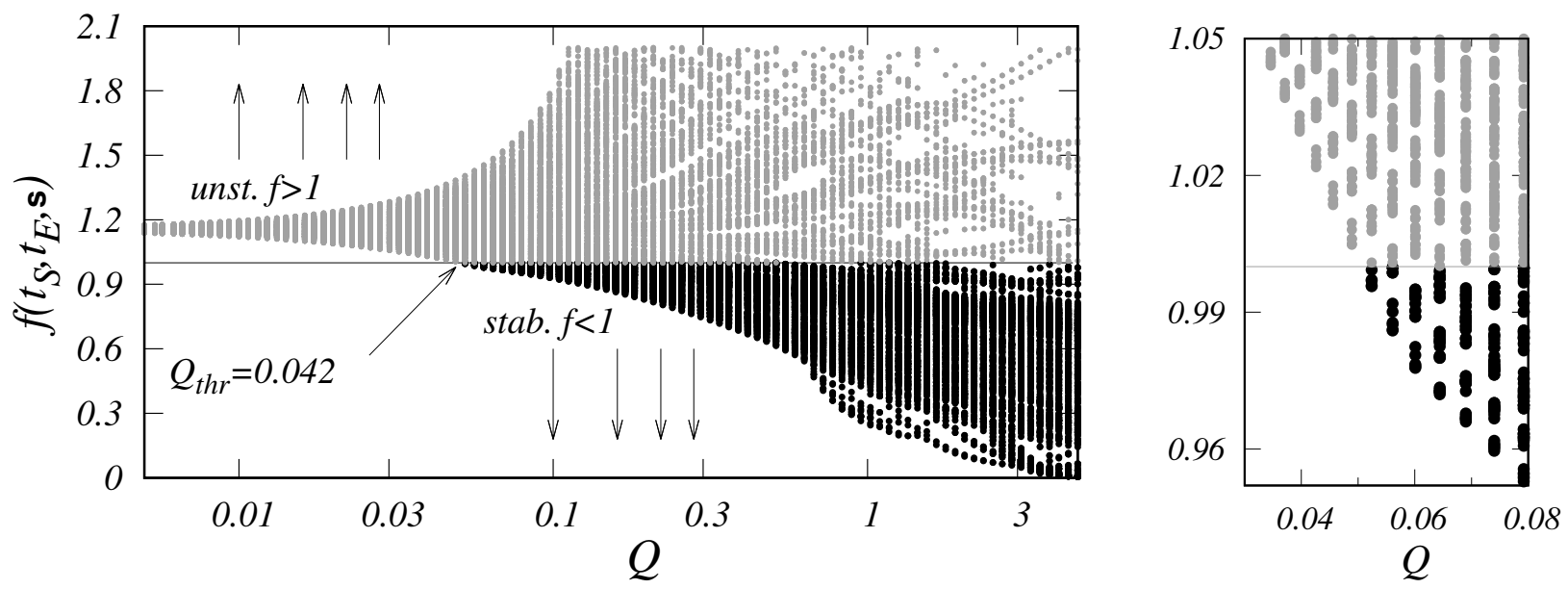

(c) $n_{0}=2$

(d) $n_{0}=2$ detail

Figure 3: The motivation for the optimization. The figure panels calculated for different constraints, $n_{0}=0$ (panels (a), (b)) and $n_{0}=2$ (panels (c), (d)) The values of the objective function $f\left(t_{S}, t_{E}, \mathbf{s}\right.$ ) are calculated for all strings $\mathbf{s} \in \Omega$ and plotted as the function of $Q$. The results reveal the existence of parametric (and constraint dependent) threshold $Q_{t h r}\left(n_{0}\right)$ above which the therapeutic effect $f()<$.1 exists when the optimization is applied. The details of the threshold neighborhoods are shown in the panels (b), (d). In all cases the stability line $f=1$ separates unstable (unst.) from stable (stab.) regions. The highly unstable region $f>2$ was removed from the plot. 

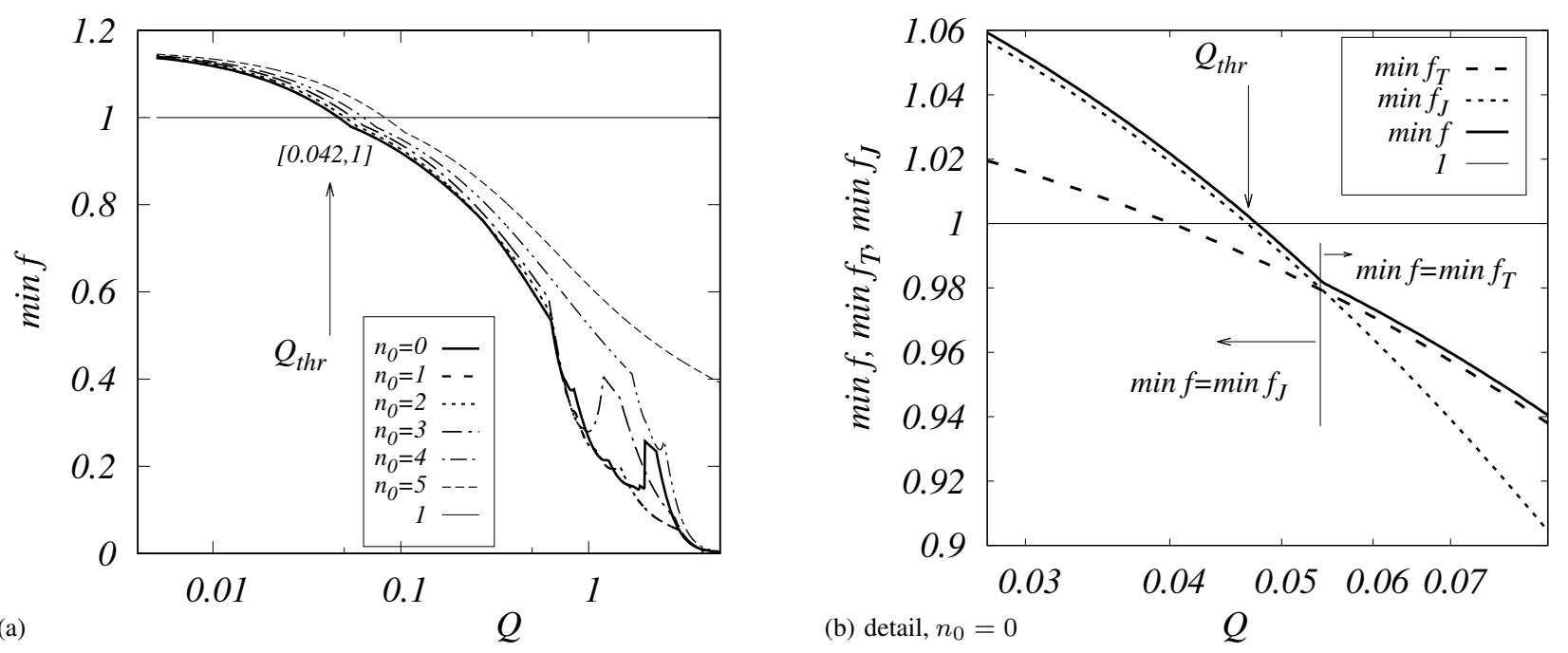

Figure 4: The plot of the optimized $f(\mathbf{s})$. Panel (a) shows the $\min f$ dependencies on $Q$ calculated for six constraints $n_{0}=0,1, \ldots, 5$. It means that strictly one discrete component among $s_{h_{C T}}, s_{g_{C C}}, s_{g_{C B}}, s_{h_{B T}}$, $s_{g_{B C}}, s_{g_{B B}}$ is requested to be nonzero in the case of $n_{0}=5$ constraint. The result that higher $n_{0}$ leads to lower efficiency of the virtual therapies (especially for high $Q$ ) is in agreement with intuitive reasoning. However, the peaked form of the $f_{\min }(Q)$ at high $Q$ lacks the intuitive understanding. It can be understood as a kind of the combined effect of the optimization, high amplitude stimuli and nonlinearities of ODEs system. Panel (b) sheds light on the problem of threshold $Q_{t h r}$ where $f\left(Q_{t h r}\right)=1$. The careful analysis shows that it is close but not identical to the point $\min f_{T}=\min f_{J}$ where the objects of the optimization [larger function from the pair $f_{T}$, $f_{J}$, see Eq.[19]] exchange. 

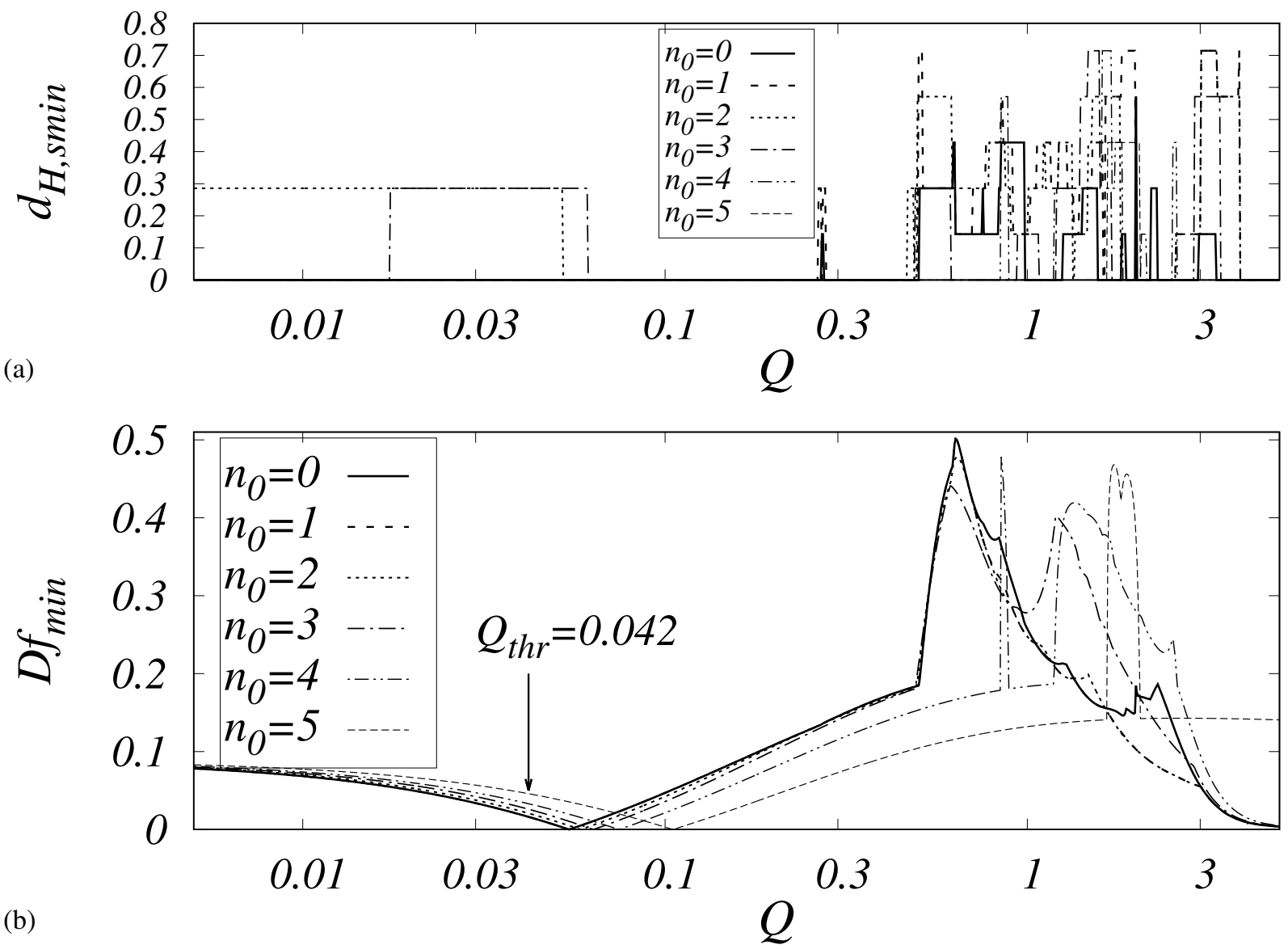

Figure 5: The panels show differences between the applied optimization criteria expressed by the functions $f_{T}$ and $f_{J}$ calculated for different $n_{0}$. The differences in the minima of $f_{T}$ and $f_{J}$ are quantified by means of the Hamming distance $d_{H, s m i n}$. The objective function values are reflected by the diversity measure $D f_{\min }$ [see panel (b)] defined by Eq.23). The subtle difference between the threshold position $Q_{t h r}\left(\right.$ for $n_{0}=0$ ) and the minimum of $D f_{\min }$ obtained for $n_{0}=0$ has been already explained in the Fig 3 . The highest diversity occurs at the large $Q$. 

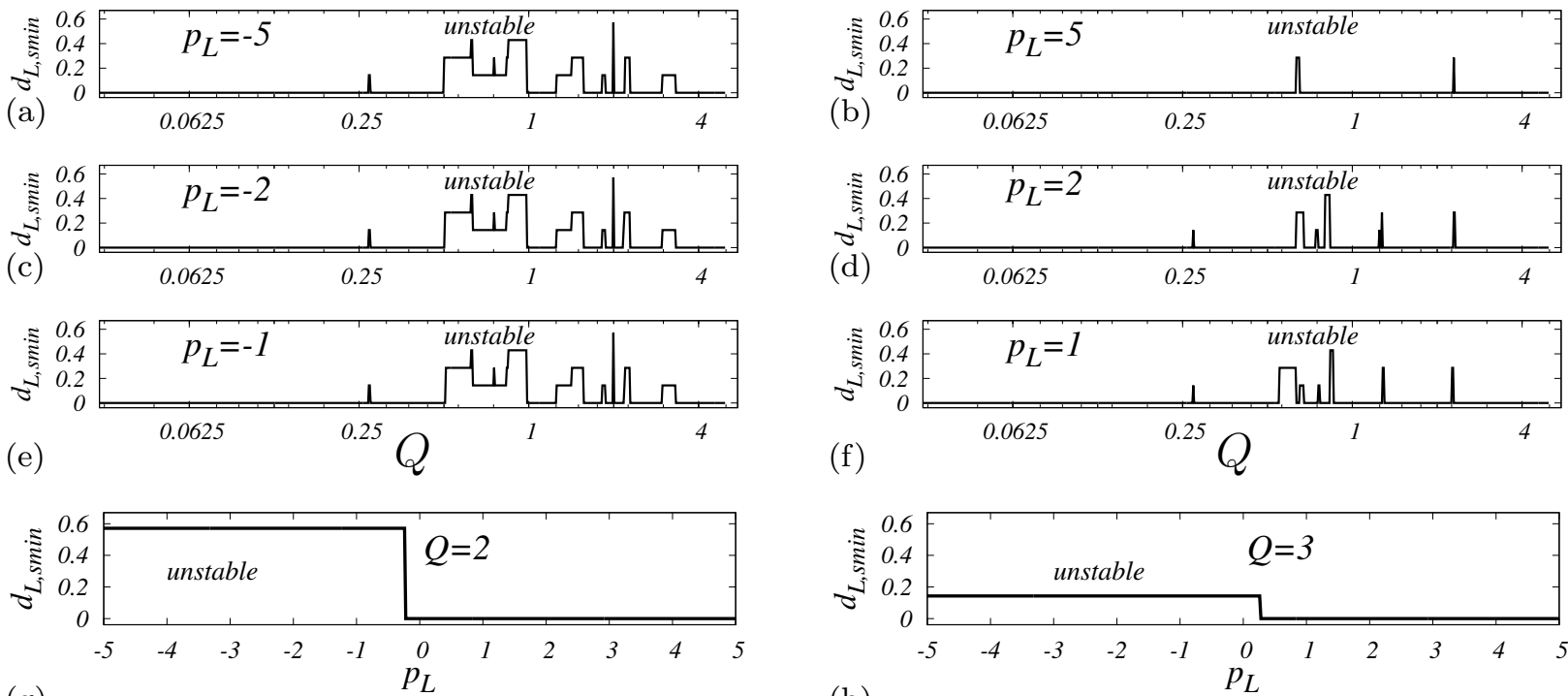

(g)

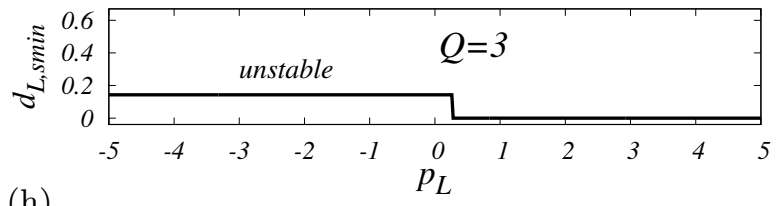

(h)

Figure 6: $Q$ and $p_{L}$ dependencies of $d_{L, s m i n}$ distances introduced to analyze relative stability of the optima of the alternative scalarizations discussed in the section 4 . The panels (b), (d), (f), (g), (h) show that increasing positive values of $p_{L}$ results in gradual vanishing of instability, in agreement with the expectation (we note that the worstcase scenario corresponds to $p_{L} \rightarrow \infty$ ). The distances calculated for the negative $p_{L}$ values $-1,-2,-5$ [panels (a) ,(c), (e)] are nearly identical to each other. Specifically, the choice of small $Q$ can guarantee higher stability, thus lowering sensitivity to the way in which the scalarization is performed. Calculated for the constraint $n_{0}=0$. 


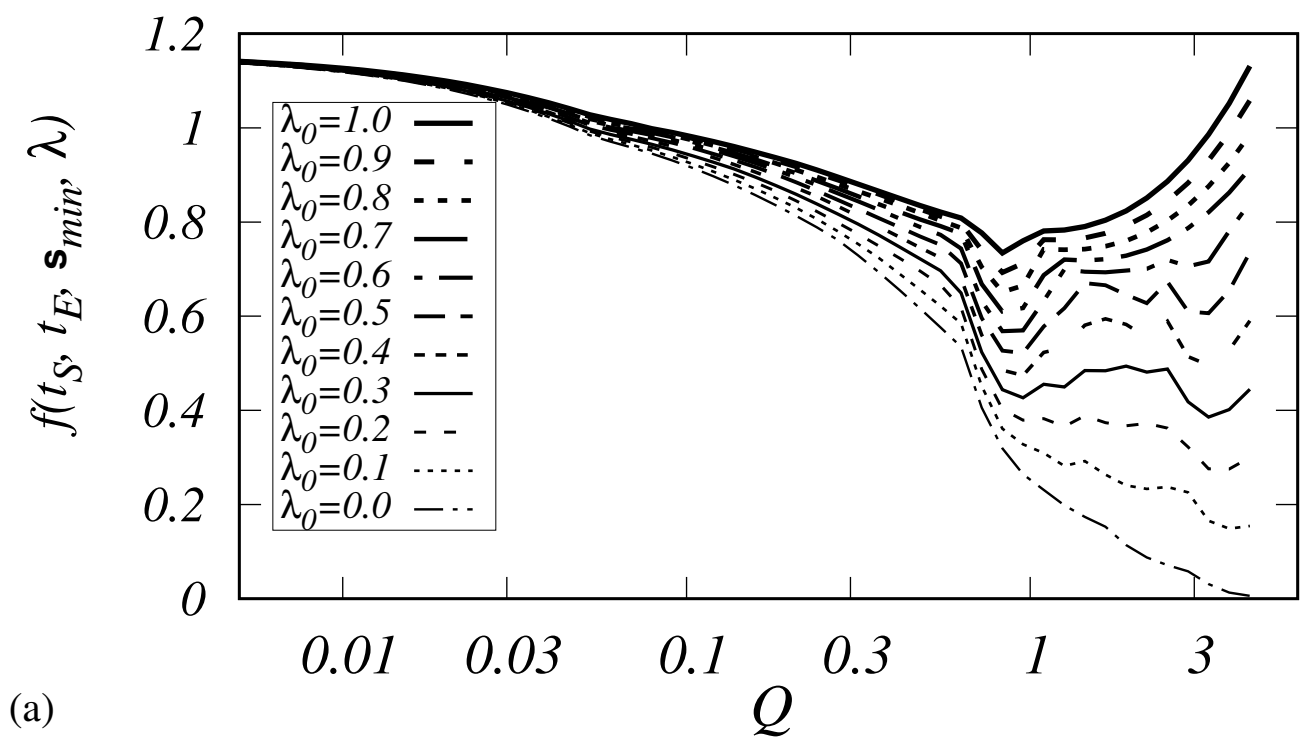

(a)
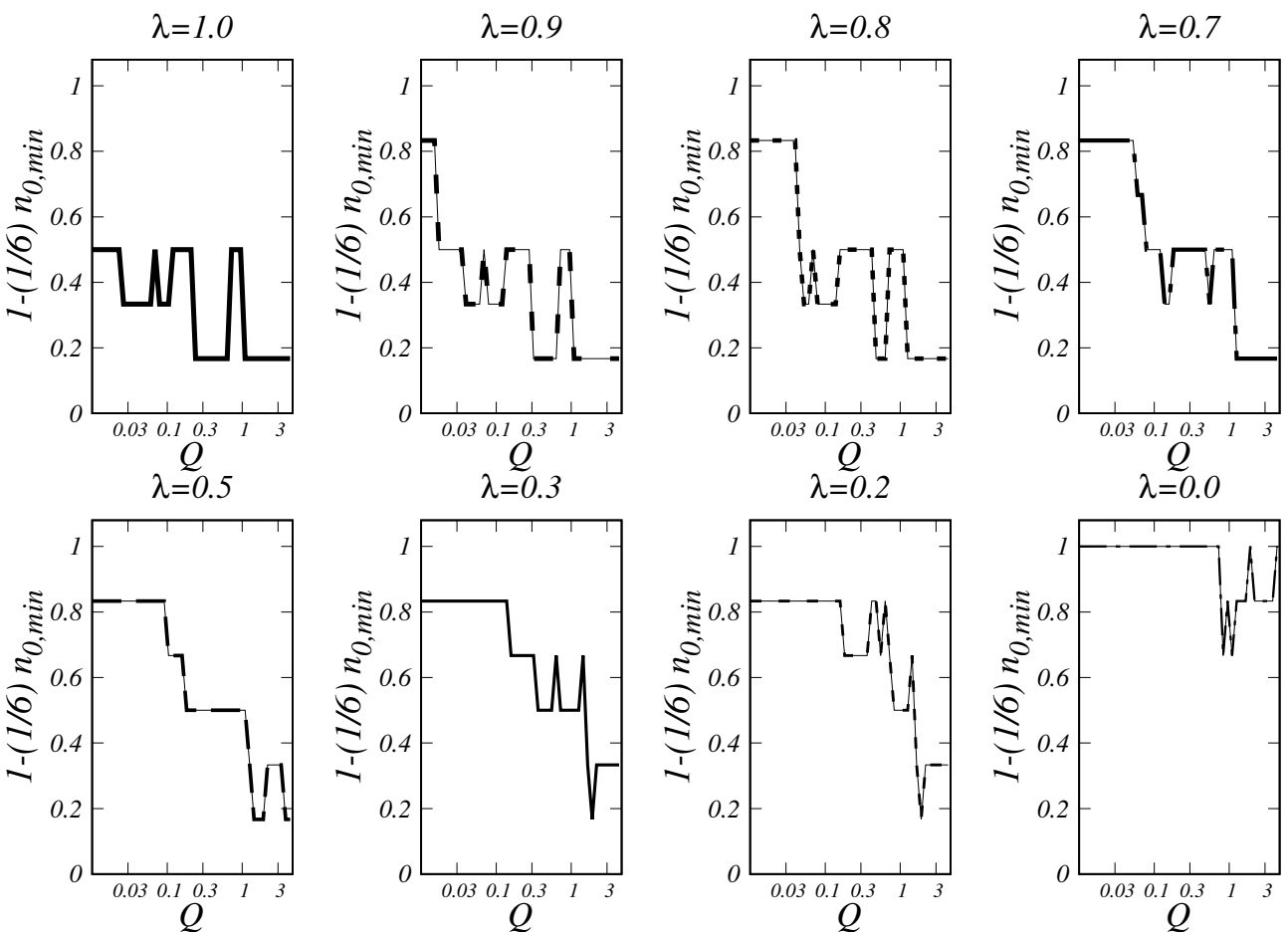

Figure 7: The $Q$-dependence of the optimized alternative objective function values $f\left(t_{S}, t_{E}, \mathbf{s}_{\min }, \lambda\right)$ [see Eq. 29]] calculated for $\lambda=0.0,0.1, \ldots, 1.0$ [panel (a)] supplemented with multiple plots of $1-(1 / 6)\left(n_{0}\right)_{\min }$ quantity [panel (b)]. The plots demonstrate how the fraction of non-zero $\mathbf{s}_{\min }$ components varies with $Q$. 


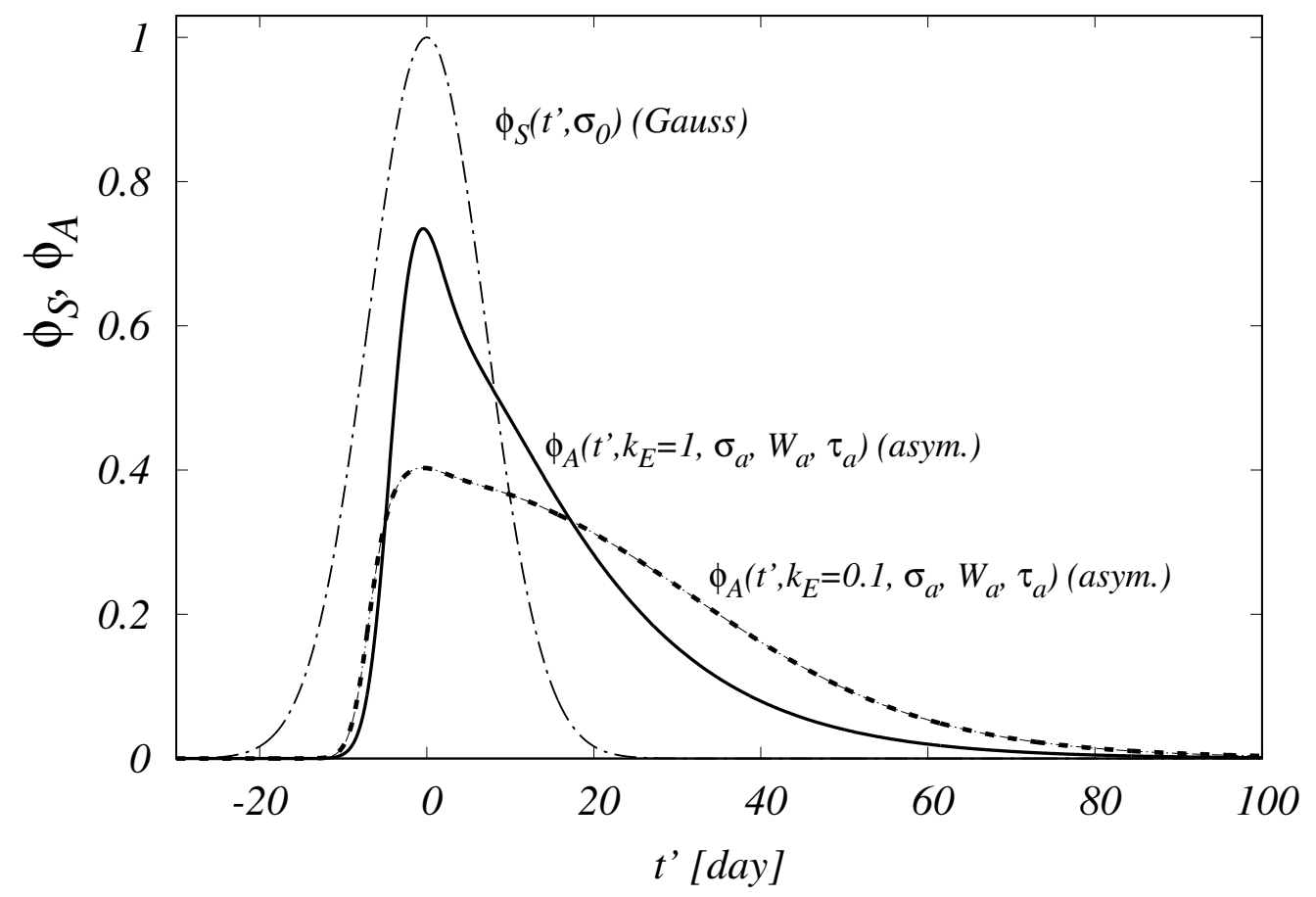

Figure 8: The figure illustrates the model of the asymmetric pulses given by Eqs. 30] and (31) which replace symmetric form [Eq.[17]]. The asymmetric pulses have been used in the numerical optimizations. 


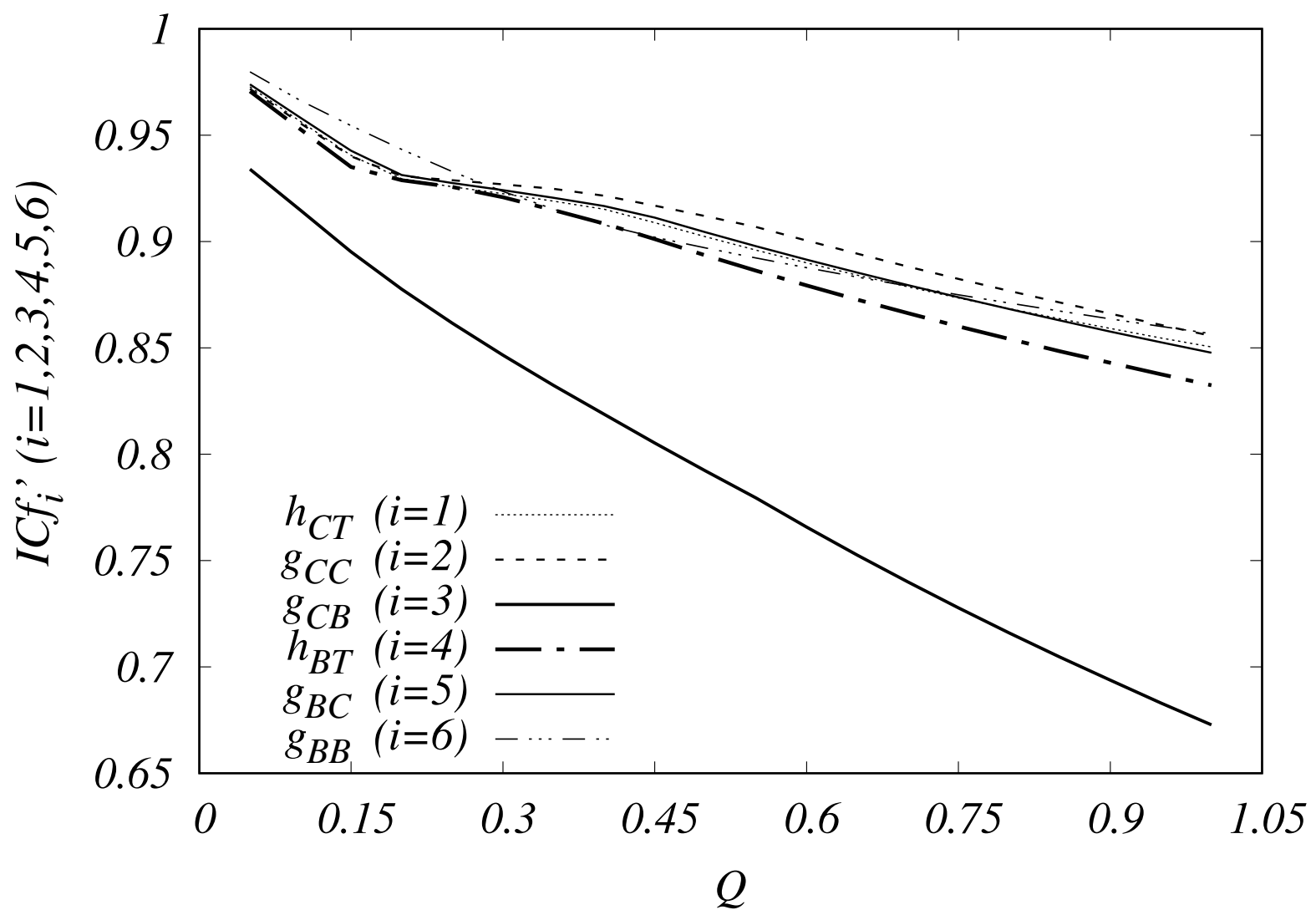

Figure 9: The comparison of the integral synergistic optimization effects of the parametric pairs; $Q$-dependence of $C I f_{i}^{\prime}, i=1,2, \ldots, 6$ calculated for the asymmetric pulses characterized by $k_{E}=1, W_{a}=2 d a y, \sigma_{a}=3 d a y$, $\tau_{a}=14$ day. 


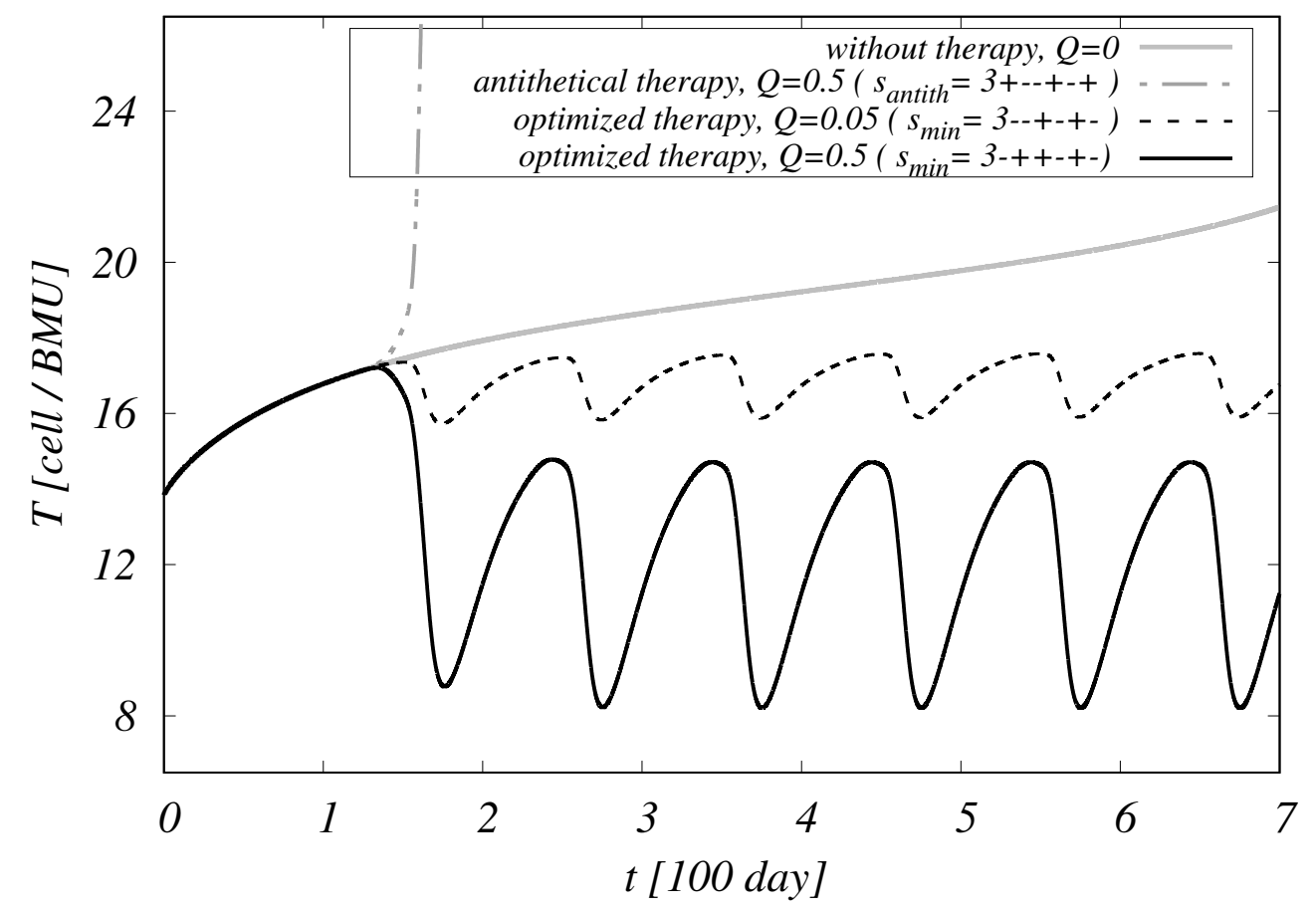

Figure 10: The illustrative treatment of the tumor by means of the periodic environmental changes. The comparison for several $Q$ values. The periodic extension of the solution obtained for $Q=0.5$ is based on the optimal string $\mathbf{s}_{\min }=[3,-1,+1,+1,-1,+1,-1]$ (optimized for the non-periodic case only). The "antithetical" virtual therapy $\mathbf{s}_{\text {antith }}=[3,+1,-1,-1,+1,-1,+1]$ (where component $\mp 1$ is replaced by \pm 1 ) has been chosen for the comparative purposes. Note that this therapy is not necessarily the worst possible therapy obtained for $Q=0.5$. The neutral [no influence of $\Psi_{B}($.$) or \Psi_{C}($.$) terms] choice calculated for Q=0$ corresponds to the tumor growth without environmental moves. 

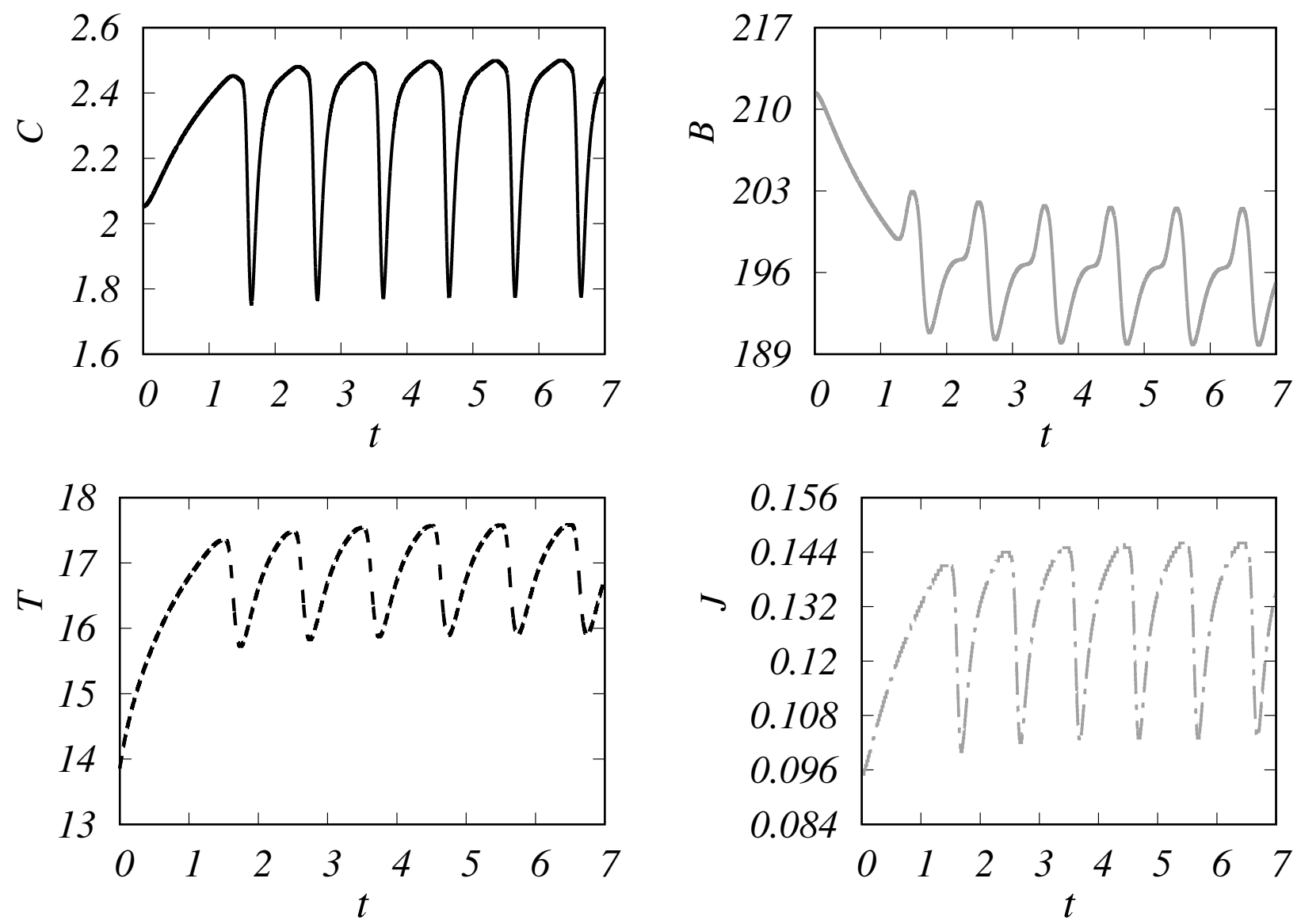

Figure 11: The onset of the periodic dynamics of the four cell populations characterized by $C, B, T, J$ (population numbers per BMU) induced by the exogenous periodic stimuli with the period $t_{E}-t_{S}$. Calculated for $Q=0.05$ and the respective string $\mathbf{s}_{\min }=[3,-1,-1,+1,-1,+1,-1]$. 

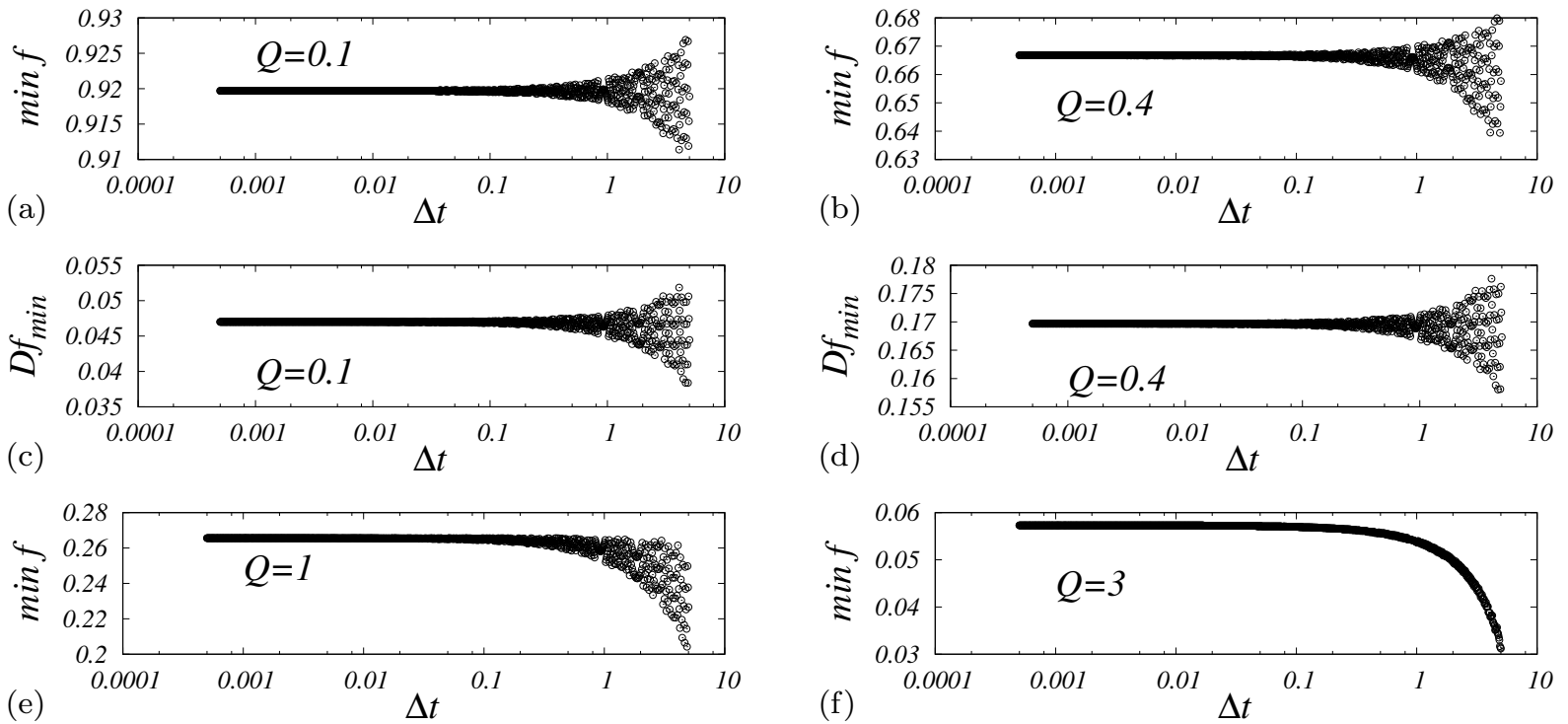

Figure 12: This figure shows results of the combined testing with the typical loss of numerical stability corresponding to the optimized outputs of the RK4 method obtained by the gradually increasing time integration step. Calculated for $n_{0}=0$ and $Q \in\{0.1,0.4,1,3\}$. Data in panels (a), (b), (e), (f) show min $f$ while (c),(d) panels show $D f_{\text {min }}$ quantity. As it is obvious from the panel (f), the instability may also have a one-sided character. Note that dependencies (a) - (e) look seemingly stochastic for $\Delta t \gtrsim 0.1$, but detailed view reveals certain regularity of patterns in this region. 